TRANSACTIONS OF THE

AMERICAN MATHEMATICAL SOCIETY

Volume 355, Number 10, Pages 4019-4043

S 0002-9947(03)03171-4

Article electronically published on June 18, 2003

\title{
FRACTAFOLDS BASED ON THE SIERPINSKI GASKET AND THEIR SPECTRA
}

\author{
ROBERT S. STRICHARTZ
}

\begin{abstract}
We introduce the notion of "fractafold", which is to a fractal what a manifold is to a Euclidean half-space. We specialize to the case when the fractal is the Sierpinski gasket SG. We show that each such compact fractafold can be given by a cellular construction based on a finite cell graph $G$, which is 3-regular in the case that the fractafold has no boundary. We show explicitly how to obtain the spectrum of the fractafold from the spectrum of the graph, using the spectral decimation method of Fukushima and Shima. This enables us to obtain isospectral pairs of nonhomeomorphic fractafolds. We also show that although SG is topologically rigid, there are fractafolds based on SG that are not topologically rigid.
\end{abstract}

\section{INTRODUCTION}

Let $K$ be a fractal $[\mathrm{F}]$. Then a fractafold $\mathcal{F}$ based on $K$ is a connected Hausdorff topological space such that every point $x$ in $\mathcal{F}$ has a neighborhood homeomorphic to a neighborhood in $K$. There is no generally agreed upon definition of "fractal", other than "I know one when I see one", but there are several well-defined classes of fractals, such as Kigami's p.c.f. (post-critically finite) self-similar fractals [Ki1]. We are interested in this class of fractals because one can do analysis on them: under certain additional hypotheses, one can construct a Laplacian $\Delta$ on $K$ and study properties of the spectrum of $\Delta$. (Of course it should be emphasized here that the Laplacian is not uniquely determined by the topology of $K$, but rather depends on certain additional geometric structures, just as the Laplacian on a manifold depends on the choice of a Riemannian metric.) One of the purposes of introducing fractafolds in this context is that we may easily extend the Laplacian from $K$ to $\mathcal{F}$, and thereby obtain a larger class of objects on which to do analysis ([B], Ki1], Ki2, $\mathrm{S} 2$ ).

We will mainly deal with the case $K=\mathrm{SG}$, the Sierpinski gasket. Recall that SG is the attractor of the ifs (iterated function system) in the plane consisting of three homotheties $\left(F_{1}, F_{2}, F_{3}\right)$ with contraction ratio $1 / 2$ and fixed-points equal to the three vetices $\left(q_{1}, q_{2}, q_{3}\right)$ of an equilateral triangle. Then SG is the unique nonempty compact set satisfying

$$
\mathrm{SG}=\bigcup_{i=1}^{3} F_{i}(\mathrm{SG})
$$

Received by the editors May 30, 2002.

2000 Mathematics Subject Classification. Primary 28A80.

This research was supported in part by the National Science Foundation, grant DMS-0140194. 
We refer to the sets $F_{i}(S G)$ as cells of level one, and by iterating (1.1) we obtain the splitting of SG into cells of higher level. Note that SG is connected, but just barely: there is a dense set of points $\mathcal{J}$, called junction points, defined by the condition that $x \in \mathcal{J}$ if and only if $U \backslash\{x\}$ is disconnected for all sufficiently small neighborhoods $U$ of $x$. It is easy to see that $\mathcal{J}$ consists of all images of $\left\{q_{1}, q_{2}, q_{3}\right\}$ under iterates of the ifs. The vertices $\left\{q_{1}, q_{2}, q_{3}\right\}$ are not junction points, but rather what we will call terminal points: if we glue together two neighborhoods of terminal points at the terminal points, we obtain a set homeomorphic to a neighborhood of a junction point. All other points in SG will be called generic points. It is clear that we may obtain a fractafold based on SG by taking a collection of copies of SG and gluing together certain pairs of terminal points (making sure that we do enough gluing to get a connected set). Note that we allow gluing of pairs of terminal points from the same copy of SG, and we allow some terminal points to remain unglued. We call this a cellular construction, and we associate with it a cell graph $G$ with one vertex for each copy of SG, and an edge for each gluing pair. Note that we allow multiple edges joining the same vertices, and loops joining a vertex to itself. We will show that every fractafold based on SG has a cellular construction. The cellular construction is not unique, but we show that there is a unique minimal one characterized by the condition that $G$ contains no triangles.

The terminal points of SG constitute its boundary in the analytic theory, although this is not a topological notion of boundary. We will therefore define the boundary of the fractafold to be all the terminal points that remain unglued in the cellular construction (intrinsically, they are the corresponding points in neighborhoods homeomorphic to neighborhoods of terminal points in SG). In particular, there are many fractafolds without boundary; in fact they are characterized by the condition that $G$ is 3-regular. These fractafolds without boundary are natural candidates for the simplest objects to work with in the analytic theory, in the same way that it is simpler to do analysis on compact manifolds without boundary than on bounded domains in Euclidean space.

Euclidean space is homogeneous, and the same is true for Euclidean half-space in the sense that any two interior points, and any two boundary points, may be interchanged by a homeomorphism. But just about the opposite is true for SG. We have topological rigidity $([\overrightarrow{B R},[\overline{D M}])$ as expressed in the following two results.

Proposition 1.1 (Topological rigidity of SG). (a) Any homeomorphism of SG onto $S G$ is one of six Euclidean isometries.

(b) Any homeomorphism of $S G$ into $S G$ is a Euclidean similarity, namely one of the above isometries followed by an iterated ifs map.

The proof of these results is an easy exercise based on the observation that there is a unique set of three junction points $\left\{p_{1}, p_{2}, p_{3}\right\}$, the midpoints of the edges joining the terminal points, with the property that $\mathrm{SG} \backslash\left\{p_{1}, p_{2}, p_{3}\right\}$ has three connected components. See $[\mathrm{BR}]$ or $[\mathrm{DM}]$ for details. A consequence of (b) is that any two homeomorphic images of SG lying in SG and intersecting in more than one point must be nested. None of these results are true for fractafolds. We will obtain a substitute for the nesting property: if $K_{1}, K_{2} \subset \mathcal{F}$ are homeomorphic to SG and $K_{1} \cap K_{2}$ is larger than a finite set, then $K_{1} \cap K_{2}$ is a finite union of copies of SG. This observation is needed in order to show that all fractafolds based on SG have a cellular construction. 
The main result of this paper is that the spectrum of $G$ determines the spectrum of $\mathcal{F}$ in an explicit fashion, for compact fractafolds based on SG without boundary. We follow the convention that the graph Laplacian on $G$ is defined by

$$
\Delta f(x)=\sum_{E_{x}}(f(y)-f(x))
$$

where $E_{x}$ denotes all edges connecting $x$ to another point (called $y$ ). Note that since $G$ is 3-regular there are exactly three terms in the sum, but some may be repeated if there are more than one edge joining two distinct points, and in the case that there is a loop joining $x$ to $x$, this counts twice in the sum but produces a net effect of zero. Also, we define the eigenvalue equation as

$$
-\Delta u=\lambda u,
$$

so we obtain a nonnegative spectrum. Explicitly, (1.3) says

$$
(3-\lambda) u(x)=u\left(y_{1}\right)+u\left(y_{2}\right)+u\left(y_{3}\right) \text { for all } x
$$

if $\left\{y_{1}, y_{2}, y_{3}\right\}$ are the three neighbors of $x$ in $G$. To pass from the spectrum of $G$ to the spectrum of $\mathcal{F}$ we use the method of spectral decimation of Fukushima and Shima [FS]. We should point out that while there are no new ideas in the extension of this method to fractafolds, the description of the spectrum in this context is perhaps more illuminating. For example, we show that certain aspects of the spectrum are controlled by whether or not $G$ is 2-colorable. Also, aside from a crude measure of size (the number of vertices in $G$ ), the bulk of the spectrum (asymptotically almost every eigenvalue) is independent of the particular fractafold.

The paper is organized as follows. In Section 2 we discuss the notion of a cellular construction in general. However, we then specialize to the case $K=\mathrm{SG}$, and for the rest of the paper we deal with only this case. We show that every fractafold based on SG has a cellular decomposition, and then discuss the cell graph associated to the cellular decomposition. This section also contains the example showing the failure of topological rigidity. The results of this section are purely topological, and do not refer to the analytic theory.

In Section 3 we begin discussion of the analytic theory, constructing a Laplacian and studying its spectrum. For the convenience of the reader we outline the definitions and basic results of Kigami [Ki1] adapted to fractafolds, but refer to [Ki1] for many of the proofs. This section also contains some simple results about the spectrum of graphs that will be needed later. These results, and some results in Section 4, are closely related to results of Shirai Shi]. Since we deal with finite graphs and keep track of spectral mutiplicities, we cannot directly quote results on infinite graphs from Shi.

In Section 4 we discuss the spectral decimation method. Again for the convenience of the reader we outline the basic results as adapted to fractafolds, but refer to [FS] for proofs. This allows us to give an explicit description of the spectrum of a compact fractafold without boundary in terms of the spectrum of the cell graph. We also give an explicit example of an isospectral pair of nonhomeomorphic fractafolds. This uses an isospectral pair of graphs that was kindly provided by Robert Brooks. In Section 5 we show how to handle the Dirichlet and Neumann spectra for fractafolds with boundary.

In Section 6 we discuss a wider class of Laplacians, which allows us to assign different "sizes" to the cells in a cellular construction, while still using the same 
standard Laplacian on SG as the model. In this generality we are not able to give an explicit description of the spectrum, but only of the portion of the spectrum associated to localized eigenfunctions supported on a single cell. Of course there are many more possibilities for defining Laplacians or elliptic type operators on fractafolds, modeled on other operators on SG. For example, one may use the same energy and different measures (self-similar or more general) [Ki1, or any of the self-similar energies in $\underline{\mathrm{Sa}}$, or even more general energies $\underline{\mathrm{MST}}$. We leave such developments for the future.

We conclude this introduction with a discussion of two directions for future work. The first involves the spectral theory of noncompact fractafolds. Teplyaev $\mathrm{T}$ ] has studied one class, which he calls infinite $S G$ 's, obtained by blowing up SG according to a procedure described in [S1], so that the structure in the large mirrors the structure in the small. For the case of such fractafolds without boundary, he shows that the spectrum is pure point, consisting of a countable set of eigenvalues with infinite multiplicity having a complete set of compactly supported eigenfunctions. In the case of an infinite SG with boundary (there is only one of these, and its boundary consists of a single point) he has similar, but less complete, results. He also discusses an example from $[\mathrm{BP}$ that falls into the class of noncompact fractafolds without boundary.

At the opposite extreme, one could consider what might be called the universal fractafold $\mathcal{U}$, whose cell graph is the 3 -regular tree $\mathcal{T}_{3}$. There is a covering map from $\mathcal{U}$ to any fractafold without boundary, determined by the covering map from $\mathcal{T}_{3}$ to any 3 -regular graph. (Although $\mathcal{U}$ is not simply connected, there is undoubtedly a way to define a relative homotopy theory that ignores small holes so that any fractafold has the same relative fundamental group as its cell graph, and $\mathcal{T}_{3}$ is simply connected.) A description of the automorphism group of $\mathcal{T}_{3}$ may be found in [F-TN], but it is so large that one usually wants to use suitable subgroups of it. Clearly each automorphism of $\mathcal{T}_{3}$ corresponds to a homeomorphism of $\mathcal{U}$, and it appears likely that the converse is true as well.

The spectrum of $\mathcal{T}_{3}$ is also described explicitly in [F-TN. It is purely absolutely continuous, and in many ways it resembles the spectrum of a noncompact Riemannian symmetric space. One can then use the spectral decimation method to create an absolutely continuous portion of the spectrum of $\mathcal{U}$. But there are also localized eigenfunctions which yield discrete eigenvalues with countable multiplicity. Presumably, together these would yield the entire spectrum, but the details have not been worked out.

The second direction for future work is to find the appropriate class of fractals for which the methods of this paper may be applied. One obstacle is that a typical p.c.f. fractal has a dense set of terminal points, so there is too much freedom in gluing cells, and there is no hope of defining a natural notion of boundary. There are, of course, many more examples in which the number of terminal points is finite, such as the Sierpinski gaskets based on higher dimensional simplices. One would also want the spectral decimation method of [Sh2] or [MT] to hold.

\section{Cellular construction}

Let $K$ be a fractal. A point $x \in K$ is called a junction point if for every sufficiently small neighborhood $U$ of $x, U \backslash\{x\}$ is disconnected and decomposes into a 
finite number of connected components. A point $y \in K$ is called terminal if a neighborhood of $y$ is homeomorphic to one of the connected components of $U \backslash\{x\}$ for some junction point $x$. One might think of terminal points as "boundary" points, but this is not always appropriate, since in many examples the set of terminal points is dense in $K$. At any rate, one can use terminal points to glue together several copies of $K$ and obtain a point with a neighborhood homeomorphic to $U$.

A cellular construction of a fractafold $\mathcal{F}$, the analog of a triangulation of a manifold, may be described by gluing together a finite or countable collection of copies $K_{j}$ of $K$ (called cells) as indicated. That is, $\mathcal{F}$ is the union of the copies $K_{j}$ with an admissible identification of points that is locally finite (only a finite number of points in each equivalence class, and only a finite number from each $K_{j}$ ) and such that each equivalence class $\left\{y_{1}, \ldots, y_{n}\right\}$ with terminal points $y_{j} \in K_{k(j)}$ (we do not prohibit $k(j)=k\left(j^{\prime}\right)$ for $j \neq j^{\prime}$ ) has the property that each $y_{j}$ has a neighborhood $U_{j}$ in $K_{k(j)}$ homeomorphic to a distinct component of $U \backslash\{x\}$ (with $x$ mapped to $y_{j}$ ) for some junction point $x$. The neighborhood system for the topology of $\mathcal{F}$ is described in the obvious way: for the identified points we take the unions of $U_{j}$ as above, as neighborhoods, while for unidentified points $z \in K_{j}$ we take neighborhoods of $z$ in $K_{j}$ not containing any terminal point.

It is easy to see that every cellular construction yields a fractafold. The converse statement is not clear in general, but it is true when $K=\mathrm{SG}$.

Theorem 2.1. Any fractafold based on $S G$ has a cellular construction.

Proof. For SG there are only three terminal points, the boundary points in $V_{0}$, and the only admissible identifications of points involve identifying two terminal points, and all such identifications are admissible. It is easy to see that any fractafold modeled on SG may be covered by copies of SG, since every point has an open neighborhood of one of three types: i) SG with two terminal points removed (terminal point neighborhood), ii) two copies of SG, each with two terminal points removed and joined at the remaining terminal points (junction point neighborhood), iii) SG with all three terminal points removed (generic point neighborhood). Moreover, if the fractafold is compact we may obtain a finite covering. Now we claim that any nonempty intersection $C \cap C^{\prime}$ of two cells in the covering must split into a finite number of subcells of $C$. To see this we split $C^{\prime}=\bigcup C_{j}^{\prime}$ into a finite number of subcells with the property that no subcell $C_{j}^{\prime}$ contains more than one terminal point of $C$. Then either $C \cap C_{j}^{\prime}$ is empty, or it consists of a single point, or $C_{j}^{\prime}$ is contained in $C$. The reason is that otherwise, removing the terminal point of $C$ would disconnect $C_{j}^{\prime}$. By the topological rigidity of $\mathrm{SG}$, any $C_{j}^{\prime}$ contained in $C$ would have to be a subcell of $C$. In the compact case we do a finite number of splittings of our finite cover of the fractafold to obtain the desired cellular construction. In the noncompact case a similar argument works; we leave the details to the reader.

From now on we consider only fractafolds based on SG.

Definition 2.2. Given a cellular construction of a fractafold, we associate to it a graph, called the cell graph, as follows: Each vertex of the graph corresponds to a cell, and two vertices are joined by one edge for each identification of two terminal points on the cells.

Note that we allow multiple edges, and edges joining a vertex to itself. Since the cellular construction of a fractafold is not unique, there are many different cell 

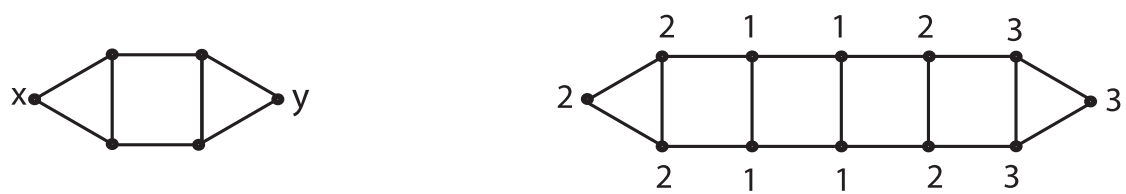

Figure 2.1. (a) The cell graph after one blowup of each of the original vertices. (b) $G(1,2)$, obtained from (a) by blowing up once on the $x$ side and twice on the $y$ side.

graphs that represent the same fractafold. We can always split a cell in a cellular construction into three cells of the next generation. On the level of the cell graph this corresponds to the basic operation we will call blowing up a point: a single vertex $v$ is replaced by three vertices $v_{1}, v_{2}, v_{3}$ joined to each other by a single edge, and for each edge joining $v$ to another vertex $v^{\prime}$ in the original graph we create an edge in the new graph joining one of the $v_{j}$ to $v^{\prime}$. There can be at most three such edges, so we make sure that we do not repeat the vertex $v_{j}$ in creating the new edges. However, if $v$ was joined to itself in the original graph, then there will be multiple edges joining some of the new points (we explicitly prohibit joining $v_{j}$ to itself). The reverse operation we call consolidating a triangle: when there are three vertices $v_{1}, v_{2}, v_{3}$ joined together in the original graph, we replace them by a single vertex, and for all other edges joining $v_{j}$ to $v^{\prime}$ we create a corresponding edge joining $v$ to $v^{\prime}$.

Any connected graph where each vertex has at most three edges (we double count an edge joining a vertex to itself) corresponds to a cellular construction of a fractafold. Compact fractafolds correspond to finite graphs. We may define an equivalence class on the set of all such graphs by repeated applications of the two basic operations. Clearly, all equivalent graphs represent the same fractafold. For a compact fractafold the converse holds as well, by essentially the same argument used to prove existence of cellular contructions. We define the boundary of a fractafold to be the set of points $x$ having a neighborhood homeomorphic to SG with $x$ mapped to a terminal point. In terms of a cellular construction, these are just the terminal points of the cells that are not identified with other points. Clearly a fractafold is without boundary if and only if its cell graph is 3-regular.

We now describe a simple example that shows that topological rigidity does not hold for fractafolds. Consider a cell graph consisting of two vertices joined by two edges. The corresponding fractafold is just two copies of SG joined at two pairs of terminal vertices (to embed this in the plane we need to distort the copies slightly from the usual presentation). We will exhibit an infinite family of homeomorphisms that fix the two boundary points. It follows that we can apply this construction locally to any cell graph containing a double edge.

If we blow up both of the vertices of the original cell graph we obtain the cell graph in Figure 2.1 (a), with two vertices of order two, called $x$ and $y$. Now iterate this process $n$ times starting with $x$ and $m$ times starting with $y$. The resulting graph is shown in Figure 2.1 (b) with $n=1$ and $m=2$, with each vertex labeled according to the number of times it has been blown up. Call the resulting graph $G(n, m)$. Clearly $G(n, m)$ is isomorphic to $G\left(n^{\prime}, m^{\prime}\right)$ if $n+m=n^{\prime}+m^{\prime}$, and the isomorphism determines a homeomorphism of the fractafold which involves identifying 

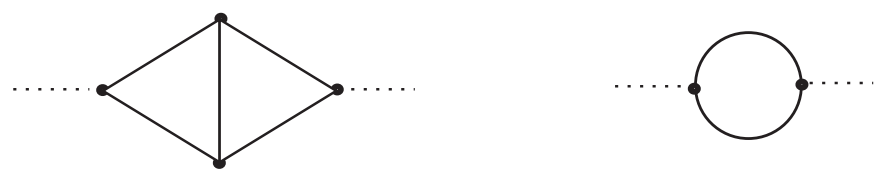

Figure 2.2. (a) The vertical edge belongs to two triangles. The dotted edges may or may not be there, and may terminate at any vertex except the two vertices on the vertical edge. (b) The configuration after consolidating either triangle.

cells of different generations. Because the difference in generations varies from cell to cell, these homeomorphisms are not isometries, and they are all distinct. The reader is encouraged to visualize these homeomorphisms as "accordian" motions of the fractafold. This example also shows that there are many different ways that two cells may intersect in a fractafold, in contrast to the trivial situation for cell intersections in the fractal.

Despite the ambiguity in "translating" from the cell graph to the fractafold, we can unambiguously define a smallest possible cell graph corresponding to a fractafold. The reason for this is that while we may be faced with choices when we apply the operation of consolidating triangles, the choices do not affect the graph we obtain. Suppose we have an edge that belongs to two triangles, like the vertical edge in Figure 2.2 (a). Whether we consolidate the left triangle or the right triangle, we obtain the configuration in Figure 2.2 (b). So each fractafold corresponds to a unique cell graph with no triangles. In particular, two fractafolds are homeomorphic if and only if their minimal cell graphs are graph isomorphic.

\section{Spectrum of the Laplacian}

Let $\mathcal{F}$ be a compact fractafold based on SG without boundary, given by a cellular construction with cell graph $G$. We will not assume that $G$ is minimal, because implicit in our definition of the Laplacian is the assumption that all cells in the construction are of equal size, while the operation of consolidating a triangle will change the size of just the consolidated cell. It might be useful for the reader to keep in mind two simple examples. The first, called the double of $S G$, has cell graph $G$ with two vertices connected by three edges; the fractafold consists of two copies of SG joined at the three terminal points. The second, called the octahedron fractafold, has cell graph $G$ equal to the complete graph with four vertices; the fractafold consists of four copies of SG placed on four faces of the regular octahedron with no common edges (each face intersects the other three in a single vertex). Of course these fractafolds are homeomorphic (in several different ways), but they will have different Laplacians in the following construction.

Although there are many different Laplacians on SG, we will only use the symmetric self-similar Laplacian of Kigami [Ki1], which we denote by $\Delta$. It is an easy matter to transfer the definition of $\Delta$ from SG to the fractafold, treating each cell as a full size SG, and using the obvious identification of a neighborhood of an identified terminal point on two copies of SG with a neighborhood of a junction point on SG. But we also present a self-contained construction as follows. 
The first step is to create a sequence of graphs $\Gamma_{0}, \Gamma_{1}, \ldots$ that will approximate the fractafold. The first of these, $\Gamma_{0}$, is simply the edge graph of $G$. The vertices $V_{0}$ of $\Gamma_{0}$ are in one-to-one correspondence with edges of $G$, and $v$ is connected to $v^{\prime}$ in $\Gamma_{0}$ if and only if the corresponding edges in $G$ meet at a $G$ vertex. (Note that if $v$ and $v^{\prime}$ correspond to a double edge in $G$, then we create two edges in $\Gamma_{0}$ connecting $v$ and $v^{\prime}$. Similarly, if $v$ corresponds to a loop in $G$, then there will be a loop in $\Gamma_{0}$ connecting $v$ to itself, and a double edge connecting $v$ to $v^{\prime}$.) If $x$ is a vertex in $G$ it has exactly three edges touching it, and the vertices of $\Gamma_{0}$ corresponding to these edges will be identified with the terminal points of the cell that $x$ represents. Each such terminal point belongs to exactly two cells, namely the two cells represented by the points in $G$ connected by the edge. This is exactly the identification of terminal points of cells dictated by the cell graph $G$. (Note that the same construction can be made to work for fractafolds with boundary if we create "virtual edges" with only one endpoint so that every vertex in $G$ has three edges. The virtual edges will then correspond to boundary points of the fractafold.)

The graph $\Gamma_{0}$ is 4-regular, since every edge in $G$ meets two other edges at each of its two endpoints, and if some of these edges coincide, then our double counting conventions will come into play. Note that each vertex $x$ in $G$ gives rise to a triangle of vertices in $\Gamma_{0}$, namely the vertices in $\Gamma_{0}$ corresponding to the three edges in $G$ that meet $x$. We call these cell triangles. Cell triangles may be degenerate: for example, $v, v$, and $v^{\prime}$ form a cell triangle if $v$ has a loop and there are two edges connecting $v$ and $v^{\prime}$. Also, the same three vertices may form two distinct cell triangles if there are double edges joining each pair of them. $\Gamma_{0}$ may also contain other triangles that are not cell triangles. Figure 3.1 shows some examples of cell graphs $G$ and their corresponding $\Gamma_{0}$ graphs.

Once we have $\Gamma_{0}$, we obtain $\Gamma_{1}$ by a process we call splitting: each cell triangle in $\Gamma_{0}$ splits into three cell triangles in $\Gamma_{1}$ by adding three new vertices at the "midpoints" of the edges of the cell triangle, as illustrated in Figure 3.2. This corresponds to splitting the cells of the fractafold into three cells of the next generation. Then $\Gamma_{k+1}$ is obtained from $\Gamma_{k}$ by the same process. Since SG is obtained from a single triangle in the limit from an infinite sequence of splittings, we obtain the fractafold from $\Gamma_{k}$ in the limit as $k \rightarrow \infty$. Note that splitting of $\Gamma_{0}$ corresponds to the operation of blowing up each of the vertices of $G$, so we have the commutative diagram

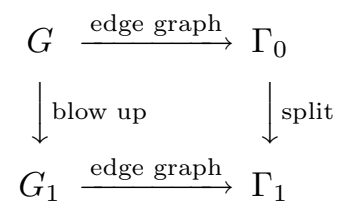

We may iterate this process, so that $G_{k}$ is the cell graph of the level $k$ cells, and $\Gamma_{k}$ is the edge graph of $G_{k}$. The vertices of $\Gamma_{k}$ may be identified with actual points on the fractafold, namely the terminal points of the cells of level $k$.

If $u$ is a real valued function on the fractafold, we may regard it as a function on each of the vertex sets $V_{k}$ of $\Gamma_{k}$ by restriction. The graph energy is defined by

$$
E_{k}(u, u)=\sum_{x \sim_{k} y}|u(x)-u(y)|^{2}
$$



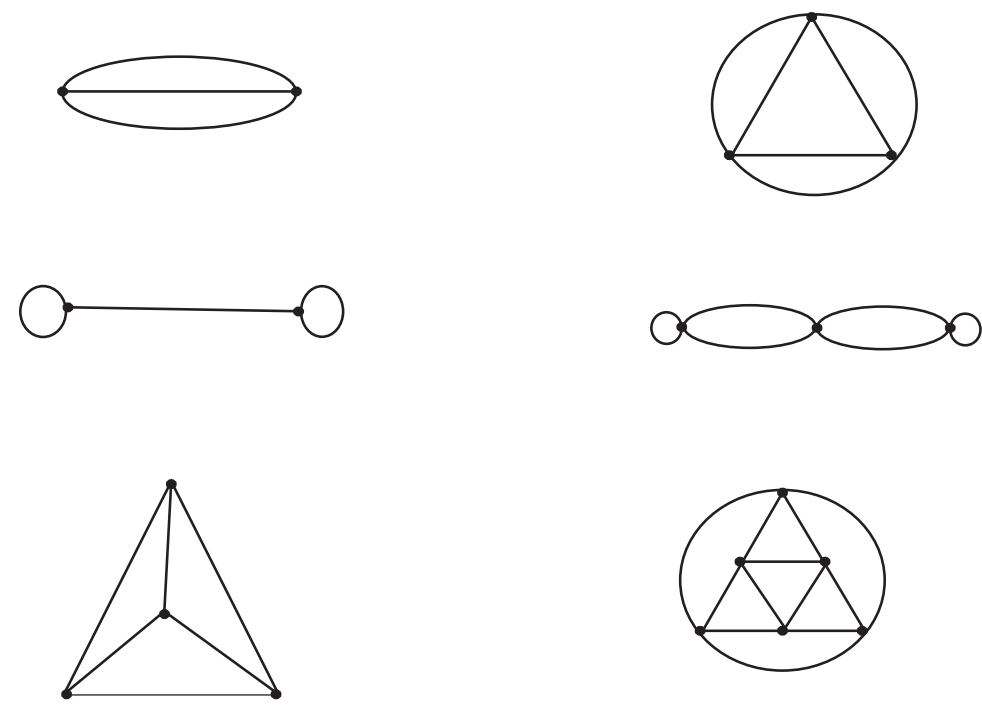

Figure 3.1. Examples of cell graphs (left) and corresponding $\Gamma_{0}$ graphs (right). Top: the double SG. Note that the three vertices of $\Gamma_{0}$ form two distinct triangles. Middle: Note that $\Gamma_{0}$ has two degenerate triangles with just two vertices. Bottom: the octahedron fractafold.
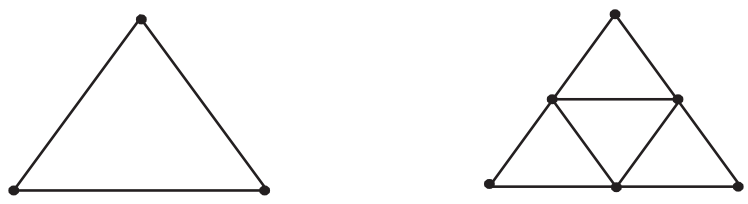

FiguRE 3.2. Splitting a triangle.

(the sum is over all edges, so if there are two edges connecting $x$ and $y$, which can only happen when $k=0$, then we double count the term). The graph Laplacian is defined by

$$
\Delta_{k} u(x)=\sum_{y \sim \sim_{k} x}(u(y)-u(x)) .
$$

Note that there are always exactly four terms in this sum. To define the analogous quantities on the fractafold we need to multiply by the appropriate renormalization factors before taking the limit. For the energy we let

$$
\mathcal{E}_{k}(u, u)=\left(\frac{5}{3}\right)^{k} E_{k}(u, u)
$$

and define

$$
\mathcal{E}(u, u)=\lim _{k \rightarrow \infty} E_{k}(u, u)
$$


The limit always exists since the sequence $\left\{\mathcal{E}_{k}(u, u)\right\}$ is monotone increasing. We define dom $\mathcal{E}$ to be the set of functions for which the limit is finite. The properties of energy on SG proved in Ki1 extend easily to fractafolds. Thus dom $\mathcal{E}$ is a dense subspace of the continuous functions, and dom $\mathcal{E}$ modulo constants becomes a Hilbert space with norm $\mathcal{E}(u, u)^{1 / 2}$. The inner product is given by

$$
\mathcal{E}(u, v)=\lim _{k \rightarrow \infty}\left(\frac{5}{3}\right)^{k} \sum_{x \sim_{k} y}(u(x)-u(y))(v(x)-v(y))
$$

with the limit existing for $u$ and $v$ in dom $\mathcal{E}$. Moreover, the factor $5 / 3$ in (3.3) and (3.4) is the only choice that doesn't lead to one of the following dead ends: all continuous functions have zero energy, or only constants have finite energy.

The Laplacian on the fractafold is defined by

$$
\Delta u(x)=\frac{3}{2} \lim _{k \rightarrow \infty} 5^{k} \Delta_{k} u(x) .
$$

Of course (3.6) only makes sense for $x$ in the vertex set $V_{*}=\bigcup_{k=0}^{\infty} V_{k}$, but $V_{*}$ is dense in the fractafold. More precisely, $u$ is in dom $\Delta$ if the limit in (3.6) is uniform. The explanation for the factor 5 in (3.6) is that it is the renormalizatoin factor $5 / 3$ for energy divided by the renormalization factor $1 / 3$ for measure. The measure $\mu$ we are using is the symmetric self-similar measure on each cell, normalized so that cells of level zero have measure one, hence cells of level $k$ have measure $3^{-k}$. The total measure of the fractafold is thus equal to the number of vertices in $G$. The factor $3 / 2$ in (3.6) is required to make the following weak formulation of $\Delta$ match up: $\Delta u=f$ for $f$ continuous if and only if $u \in \operatorname{dom} \mathcal{E}$ and

$$
-\mathcal{E}(u, v)=\int f v d \mu \text { for all } v \in \operatorname{dom} \mathcal{E} .
$$

The equivalence of (3.6) and (3.7) is proved in Ki1 for SG (actually, it is necessary to assume that $v$ vanishes on the boundary in that case) and the proof extends easily to fractafolds (see also [Ki2]). The weak formulation allows us to define the $L^{2}$ domain $\operatorname{dom}_{L^{2}} \Delta$ by (3.7) for $f \in L^{2}(d \mu)$, and then $\Delta$ becomes a self-adjoint operator with compact resolvant, hence discrete spectrum.

If we fix a cell $C$ and a terminal point $x$ in $C$, we can define the normal derivative $\partial_{n} u(x)$ of $u$ with respect to $C$ by

$$
\partial_{n} u(x)=\lim _{k \rightarrow \infty}\left(\frac{5}{3}\right)^{k}\left(2 u(x)-u\left(y_{k}\right)-u\left(z_{k}\right)\right)
$$

where $y_{k}$ and $z_{k}$ are the neighboring vertices of level $k$ to $x$ in $C$. If $u \in \operatorname{dom} \Delta$, then the normal derivatives exist, and satisfy the following matching condition: at an identified terminal point, the sum of the normal derivatives with respect to the two cells vanishes. In fact, the matching conditions provide necessary and sufficient conditions for gluing together local solutions $\Delta u_{i}=f_{i}$ on $C_{i}$ to obtain a global solution $\Delta u=f$ (here we assume that the glued functions $u$ and $f$ are continuous).

The spectrum of the Laplacian on SG (with Dirichlet or Neumann boundary conditions) was described exactly by Fukushima and Shima [FS, [Sh1] (see also [DSV], [MT], [T] and [GRS] for further elaborations) based on the method of spectral decimation Sh2. Our goal is to give an analogous description for fractafolds. We note that SG itself is a fractafold with boundary. However, if we consider the double 
of SG, its spectrum is exactly the union of the Dirichlet and Neumann spectra for SG, as the Dirichlet and Neumann eigenfunctions on SG are just the restrictions of odd and even eigenfunctions on the double. Moreover, it is easy enough to decide which eigenfunctions on the double are odd and even. Thus the spectral theory on the double is equivalent to the two spectra on SG. The same considerations hold for any fractafold $\mathcal{F}$ with boundary, as shown in Section 5 .

We will show that the spectrum of the Laplacian on $G$ determines the spectrum of $\Delta_{0}$ on $\Gamma_{0}$, and then via spectral decimation it determines the spectrum of $\Delta$ on the fractafold.

Lemma 3.1. The spectrum of the Laplacian on $G$ contains 6 if and only if $G$ is 2 -colorable, in which case 6 has multiplicity one.

Proof. If $G$ is 2-colorable, then taking $u(x)= \pm 1$ according to the color of $x$ yields an eigenfunction with eigenvalue 6 . Conversely, suppose $u$ is an eigenfunction, $-\Delta u(x)=\lambda u(x)$, and let $x_{0}$ be a vertex where $|u(x)|$ attains its maximum value. Without loss of generality, $u\left(x_{0}\right)=1$. Since $G$ is 3 -regular, we have $\lambda \leq 6$ with equality if and only if $u(y)=-1$ at all three vertices connected to $x_{0}$. Continuing in this manner, we find that $\lambda=6$ implies $u(x)= \pm 1$ for every vertex, with signs alternating for connected vertices. This shows that $G$ is 2 -colorable. Since the 2 -coloring is unique, up to color reversal, it follows that the multiplicity of 6 is one.

Lemma 3.2 (cf. Theorem 1.2 of [Shi]). If $\lambda \neq 6$, then $\lambda$ is in the spectrum of the Laplacian on $G$ if and only if it is in the spectrum of the Laplacian on $\Gamma_{0}$, with equal multiplicities.

Proof. Let $u$ denote a function on the vertices of $G$, and let $f$ denote a function on the vertices of $\Gamma_{0}$. Consider the operator $T u=f$ defined by $f(v)=u(x)+u(y)$ when $v$ corresponds to an edge connecting $x$ to $y$ in $G$. The kernel of $T$ is just the space of 6-eigenvectors described in Lemma 3.1. If $-\Delta u=\lambda u$ on $G$ with $\lambda \neq 6$, then $f=T u$ is nonzero, and it is easy to see that $-\Delta f=\lambda f$ at $v \in \Gamma_{0}$ simply by adding the eigenvalue equations for $u$ at $x$ and $y$. This shows that the multiplicity of $\lambda$ in the $\Gamma_{0}$ spectrum is at least the multiplicity of $\lambda$ in the $G$ spectrum.

On the other hand, consider the operator $S f=u$ defined by $u(x)=f\left(v_{1}\right)+$ $f\left(v_{2}\right)+f\left(v_{3}\right)$ where $v_{1}, v_{2}, v_{3}$ correspond to the edges that meet $x$. Suppose $f$ belongs to the kernel of $S$. By adding $u(x)=0$ and $u(y)=0$ at the vertices in $G$ joined by the edge $v$, we obtain $-\Delta f(v)=6 f(v)$. Thus the kernel of $S$ is exactly the 6-eigenspace. Now if $-\Delta f=\lambda f$ for $\lambda \neq 6$, we obtain $-\Delta u(x)=\lambda u(x)$ by adding the equations $-\Delta f\left(v_{j}\right)=\lambda f\left(v_{j}\right)$ for the three $v_{j}$ corresponding to edges meeting $x$. This yields the multiplicity comparison in the other direction.

Let $N$ denote the number of vertices in $G$. Note that $N$ must be even. Then $\Gamma_{0}$ has $\frac{3}{2} N$ vertices. It follows that $m_{0}(6)$, the multiplicity of 6 in the $\Gamma_{0}$ spectrum, must be $\frac{1}{2} N+1$ if $G$ is 2-colorable, and $\frac{1}{2} N$ if $G$ is not 2-colorable.

In order to use the spectral decimation method, we need to find the multiplicities of the special eigenvalues 2, 5 and 6 for each of the graphs $\Gamma_{k}$. Moreover, we will also explicitly give a basis for the corresponding eigenspaces for all $k \geq 1$. The case $\lambda=6$ is quite easy to do using our previous lemmas. Since $G_{k}$ is not 2 -colorable for any $k \geq 1,6$ is not in the spectrum of $G_{k}$, and the multiplicity $m_{k}(6)$ in $\Gamma_{k}$ must be exactly the difference in the total number of eigenvalues of $\Gamma_{k}$ and $G_{k}$, since the 


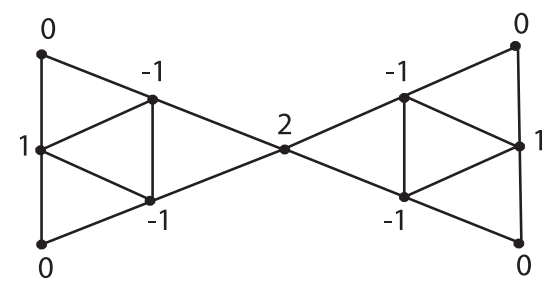

Figure 3.3. The values of a 6 -eigenfunction on two adjacent $(k-1)$-cells.

other eigenvalues are paired off. But the number of eigenvalues is just the number of vertices, and $G_{k}$ has $3^{k} N$ vertices while $\Gamma_{k}$ has $\frac{3}{2}\left(3^{k} N\right)$, so $m_{k}(6)=\frac{1}{2} 3^{k} N$ for $k \geq 1$. Note that $\frac{1}{2} 3^{k} N$ is exactly the number of vertices $V_{k-1}$ of $\Gamma_{k-1}$. It is easy to construct a 6 -eigenfunction on $\Gamma_{k}$ which takes the value one on a given vertex of $V_{k-1}$ and zero on all the other vertices of $V_{k-1}$, so these clearly form a basis of the 6 -eigenspace. Figure 3.3 shows the values of the eigenfunction on the vertices of the two $(k-1)$-cells that intersect at the given vertex; the function is zero elsewhere, so the adjacent $(k-1)$-cells form the support of the function.

We consider next the case $\lambda=2$.

Lemma 3.3. 2 is an eigenvalue of the Laplace $\Delta_{1}$ on $\Gamma_{1}$ if and only if $G$ is 2 -colorable, in which case it has multiplicity one. 2 is not an eigenvalue of the Laplacian $\Delta_{k}$ on $\Gamma_{k}$ for any $k \geq 2$.

Proof. Suppose $G$ is 2-colorable, and let $u$ be the 6-eigenfunction on $G$ constructed in Lemma 3.1. When we create the blowup $G_{1}$, define $u_{1}$ on $G_{1}$ by $u_{1}(y)=u(x)$ if $y$ is any of the three vertices associated to $x$ in the blowup. If, say, $u_{1}(y)= \pm 1$, then $y$ has two neighbors in $G_{1}$ where $u_{1}= \pm 1$ (the other two blowup points associated to $x$ ), and one neighbor where $u_{1}=\mp 1$, so $-\Delta_{1} u_{1}(y)=2 u_{1}(y)$. Conversely, if $u_{1}$ is a 2-eigenfunction on $\Gamma_{1}$, define $u(x)=\frac{1}{3}\left(u_{1}\left(y_{1}\right)+u_{1}\left(y_{2}\right)+u_{1}\left(y_{3}\right)\right)$ on $G$, where $x$ blows up to $\left(y_{1}, y_{2}, y_{3}\right)$. We claim that $u$ is not identically zero. Indeed, suppose to the contrary that $u \equiv 0$. Since $u_{1}$ is not identically zero, choose $y_{1}$ so that $\left|u_{1}\left(y_{1}\right)\right|$ attains the maximum value of $\left|u_{1}\right|$. But $u_{1}\left(y_{1}\right)+u_{1}\left(y_{2}\right)+u_{1}\left(y_{3}\right)=0$ and $-\Delta_{1} u_{1}\left(y_{1}\right)=3 u_{1}\left(y_{1}\right)-u_{1}\left(y_{2}\right)-u_{1}\left(y_{3}\right)-u_{1}\left(z_{1}\right)=2 u_{1}\left(y_{1}\right)$, where $z_{1}$ is the other vertex in $G_{1}$ joined to $y_{1}$. This says $u_{1}\left(z_{1}\right)=2 u_{1}\left(y_{1}\right)$, contradicting the maximality of $\left|u_{1}\left(y_{1}\right)\right|$.

Now it is easy to see that $u$ is a 6 -eigenvector on $G$. We simply add twice the 2-eigenvalue equation for $u_{1}$ at the points $y_{1}, y_{2}, y_{3}$ together with the 2-eigenvalue equation for $u_{1}$ at $z_{1}, z_{2}, z_{3}$, where $z_{j}$ denotes the other point connected to $y_{j}$ in $G_{1}$. The result is the 6 -eigenvalue equation for $u$ at $x$. Thus we have shown $m_{G_{1}}(2)=$ $m_{G}(6)$, and $m_{G}(6)=1$ if $G$ is 2 -colorable, zero otherwise by Lemma 3.1. But $m_{G_{1}}(2)=m_{1}(2)$ by Lemma 3.2. For $k \geq 2$ we have $m_{k}(2)=m_{G_{k}}(2)=m_{G_{k-1}}(6)$, but $m_{G_{k-1}}(6)=0$ because $G_{k-1}$ is not 2-colorable (it contains triangles).

In the case that $G$ is 2-colorable, we can describe the 2-eigenvector on $\Gamma_{1}$ explicitly as follows: $u$ is zero on all the old vertices $V_{0}$ of $\Gamma_{0}$, and on the new vertices $V_{1} \backslash V_{0}, u= \pm 1$ depending on the color of the cell the vertex is in.

For the case $\lambda=5$ we use a slightly different strategy. We first construct $3^{k-1} \frac{N}{2}+$ 1 linearly independent 5-eigenfunctions on $G_{k}$ and $\Gamma_{k}$. This will show $m_{k}(5) \geq$ 
$3^{k-1} \frac{N}{2}+1$. In the next section we will use a counting argument to show that we actually have equality.

Lemma 3.4. G has $\frac{N}{2}+1$ independent (in the sense of homology) simple cycles.

Proof. The proof is by induction on the even number $N$. When $N=2$ there are only two possibilities, shown as the first two examples in Figure 3.1, and we see by inspection the two simple cycles. (In fact the first example has three distinct simple cycles, but only two are independent.)

For the induction step, we assume the result is true for 3-regular graphs with $N-2$ vertices, and consider $G$ with $N$ vertices. Suppose first that $G$ contains a loop on the vertex $x$. Then $x$ must connect to exactly one other vertex $y$. Form a graph $G^{\prime}$ by removing $x$ and $y$ from $G$, and add an edge connecting $z_{1}$ and $z_{2}$, the two other points connected to $y$. (It may happen that $z_{1}=z_{2}$.) Note that $G^{\prime}$ is connected and 3-regular, so by the induction hypothesis it has $N / 2$ independent simple cycles. Each of these cycles corresponds to a cycle in $G$ (if the cycle in $G^{\prime}$ contains the edge joining $z_{1}$ and $z_{2}$, then the corresponding cycle in $G$ contains the edges joining $z_{1}$ and $z_{2}$ to $y$ ). In addition we have the loop on $x$, which is clearly independent of the others.

Next consider the case that $G$ has no loops. It is easy to see that $G$ must contain at least one simple cycle (keep moving without backtracking until you return to a vertex). Let $x$ and $y$ be adjacent vertices that lie on a simple cycle. Form a graph $G^{\prime}$ by removing $x$ and $y$ from $G$, and adding edges connecting $x^{\prime}$ to $x^{\prime \prime}$ and $y^{\prime}$ to $y^{\prime \prime}$ where $x^{\prime}$ and $x^{\prime \prime}$ are the other vertices connected to $x$ in $G$, and $y^{\prime}$ and $y^{\prime \prime}$ are the other vertices connected to $y$ in $G$ (again there may be some coincidences among the points $\left.x^{\prime}, x^{\prime \prime}, y^{\prime}, y^{\prime \prime}\right)$. It is clear that $G^{\prime}$ is connected and 3-regular, and has one fewer independent simple cycles.

Given a simple cycle $x_{0}, x_{1}, x_{2}, \ldots, x_{n}=x_{0}$ in $G$, we create a 5 -eigenfunction on $G_{1}$ as follows. Let $x_{j}^{\prime}, x_{j}^{\prime \prime}, x_{j}^{\prime \prime \prime}$ denote the triangle in $G_{1}$ that replaces $x_{j}$, chosen so that $x_{j-1}^{\prime \prime}$ and $x_{j}^{\prime}$ are adjacent vertices in $G_{1}$ for $1 \leq j \leq n$. Then $x_{0}^{\prime}, x_{0}^{\prime \prime}, x_{1}^{\prime}, x_{1}^{\prime \prime}, \ldots, x_{n-1}^{\prime}, x_{n-1}^{\prime \prime}, x_{n}^{\prime}=x_{0}^{\prime}$ is an even cycle in $G_{1}$. We set $u\left(x_{j}^{\prime}\right)=1$, $u\left(x_{j}^{\prime \prime}\right)=-1$, and let $u=0$ at all other vertices. Then the 5 -eigenvalue equation holds at all vertices of the cycle, since each vertex has two neighbors of the opposite sign. It also holds trivially at the points $x_{j}^{\prime \prime \prime}$ since $u\left(x_{j}^{\prime \prime \prime}\right)=0$ while $u\left(x_{j}^{\prime}\right)+u\left(x_{j}^{\prime \prime}\right)=0$, and even more trivially at all other points where every term is zero.

It is clear from the construction that independent simple cycles yield independent eigenfunctions. We can apply the same reasoning to the graphs $G_{k}$ also. This yields

$$
m_{5}\left(G_{k}\right)=m_{5}\left(\Gamma_{k}\right) \geq 3^{k-1} \frac{N}{2}+1
$$

We may also describe the 5-eigenfunctions on $\Gamma_{k}$ directly as follows. The simple cycle in $G_{k-1}$ may be interpreted as a cycle of $(k-1)$-cells in $\Gamma_{k-1}$ connected at two terminal points each. Each of these cells splits into three $k$-cells as in Figure 3.2. We assign alternating values \pm 1 to the new vertices that run parallel to the connecting old vertices in the cycle. All other vertices have value zero. An example is shown in Figure 3.4. 


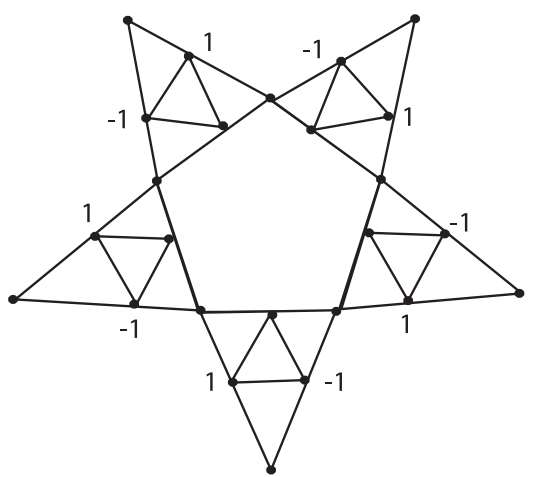

Figure 3.4. A 5-eigenfunction on $\Gamma_{k}$ associated to a simple cycle of length 5 in $G_{k-1}$.

\section{Spectral Decimation}

In this section we show how the spectrum of $G$ determines the spectrum of the fractafold. We denote by $\left\{\mu_{j}\right\}, j=0, \ldots, N_{0}$, the distinct eigenvalues of $G$ (in increasing order) except for 6 , with multiplicities $m_{G}\left(\mu_{j}\right)$. (Of course $\mu_{0}=0$ with $m(0)=1$.) We have

$$
\sum_{j=0}^{N_{0}} m_{G}\left(\mu_{j}\right)=\left\{\begin{array}{l}
N-1 \text { if } G \text { is 2-colorable } \\
N \text { if } G \text { is not 2-colorable. }
\end{array}\right.
$$

The spectrum of $\Gamma_{0}$ is then $\left\{\mu_{0}, \ldots, \mu_{N_{0}}, 6\right\}$ with multiplicities

$$
\left\{\begin{array}{l}
m_{0}\left(\mu_{j}\right)=m_{G}\left(\mu_{j}\right), \\
m_{0}(6)=\left\{\begin{array}{l}
\frac{1}{2} N+1 \text { if } G \text { is 2-colorable, } \\
\frac{1}{2} N \text { if } G \text { is not 2-colorable. }
\end{array}\right.
\end{array}\right.
$$

We now describe the spectral decimation algorithm to relate the spectra of $\Gamma_{k}$ and $\Gamma_{k-1}$. Let

$$
p(x)=x(5-x),
$$

and let

$$
\varphi_{ \pm}(x)=\frac{5 \pm \sqrt{25-4 x}}{2}
$$

be the two inverses (we will only deal with the interval $0 \leq x \leq 6$ where the square root is well-defined).

Proposition 4.1. Let $u$ be a $\lambda$-eigenfunction on $\Gamma_{k}$ for $\lambda \neq 2,5,6$. Then the restriction of $u$ to $\Gamma_{k-1}$ is a $p(\lambda)$-eigenfunction, and if $x, y, z$ are the terminal vertices of $a(k-1)$-cell in $\Gamma_{k-1}$ and $w$ is the new vertex in $\Gamma_{k}$ connected to $x$ and $y$, then

$$
u(w)=\frac{4-\lambda}{(2-\lambda)(5-\lambda)}(u(x)+u(y))+\frac{2}{(2-\lambda)(5-\lambda)} u(z) .
$$

Conversely, given a $p(\lambda)$-eigenfunction $u$ on $\Gamma_{k-1}$ with $\lambda \neq 2,5,6$, if we use (4.5) to extend $u$ to $\Gamma_{k}$, then we obtain a $\lambda$-eigenfunction on $\Gamma_{k}$. 
This result is proved in [FS] for SG, and the proof may also be found in DSV]. The argument is entirely local, so the extension to fractafolds is immediate.

Note that $p(2)=p(3)=6$ and $p(5)=p(0)=0$. Thus 6 -eigenfunctions on $\Gamma_{k-1}$ extend to 3 -eigenfunctions on $\Gamma_{k}$ but not 2-eigenfunctions. Similarly, 0eigenfunctions on $\Gamma_{k-1}$ extend to 0 -eigenfunctions on $\Gamma_{k}$ but not 5 -eigenfunctions, but the only 0 -eigenfunctions are the constants. Note that if $\lambda \neq 2,5,6$, then the proposition implies that $0 \leq \lambda<5$, because if $\lambda>5$, then $p(\lambda)<0$. We use the convention of placing eigenvalues in a box if they are present only in the case that $G$ is 2-colorable.

Theorem 4.2 (cf. Theorem 1.8 of [Shi] $)$. Let $\left\{\lambda_{0}^{(k-1)}=0, \lambda_{1}^{(k-1)}, \ldots, \lambda_{n}^{(k-1)}, 6\right\}$ be the spectrum of $\Gamma_{k-1}$ for $k \geq 1$ in increasing order with multiplicities $m_{k-1}\left(\lambda_{j}^{(k-1)}\right)$. Then the spectrum of $\Gamma_{k}$ in increasing order is

$$
\left\{0, \varphi_{-}\left(\lambda_{1}^{(k-1)}\right), \ldots, \varphi_{-}\left(\lambda_{n}^{(k-1)}\right), 2,3, \varphi_{+}\left(\lambda_{n}^{(k-1)}\right), \ldots, \varphi_{+}\left(\lambda_{1}^{(k-1)}\right), 5,6\right\},
$$

with 2 present only when $k=1$ if $G$ is 2 -colorable (and then $m_{1}(2)=1$ ), with multiplicities

$$
\left\{\begin{array}{l}
m_{k}(0)=1, \\
m_{k}\left(\varphi_{ \pm}\left(\lambda_{j}^{(k-1)}\right)\right)=m_{k-1}\left(\lambda_{j}^{(k-1)}\right), 1 \leq j \leq n, \\
m_{k}(3)=m_{k-1}(6), \\
m_{k}(5)=\frac{1}{2} 3^{k-1} N+1, \\
m_{k}(6)=\frac{1}{2} 3^{k} N .
\end{array}\right.
$$

Proof. Observe that $\varphi_{-}$is increasing and $\varphi_{+}$is decreasing, and $0<\varphi_{-}(x)<2$ and $3<\varphi_{+}(x)<5$ if $0<x<5$, so we have the correct order for the $\Gamma_{k}$ spectrum. The proposition explains the appearance of $\varphi_{ \pm}\left(\lambda_{j}^{(k-1)}\right)$ with multiplicity $m_{k-1}\left(\lambda_{j}^{(k-1)}\right)$, and also $3=\varphi_{+}(6)$ with multiplicity $m_{k-1}(6)$. The eigenvalue 0 corresponds to the constants alone. We have explained the multiplicities of 2 and 6 in the previous section, and we have shown $m_{k}(5) \geq \frac{1}{2} 3^{k-1} N+1$. To show that we have equality we just have to count.

The sum of the multiplicities for $\Gamma_{k-1}$ is $\frac{1}{2} 3^{k} N$, and for $\Gamma_{k}$ it is $\frac{1}{2} 3^{k+1} N$. We give the argument for the case $k \geq 2$, or $k=1$ and $G$ is not 2-colorable. (The remaining case is quite similar.) Then $m_{k-1}(0)=1$ and $m_{k-1}(6)=\frac{1}{2} 3^{k-1} N$, so

$$
\sum_{j=1}^{n} m_{k-1}\left(\lambda_{j}^{(k-1)}\right)=\frac{1}{2} 3^{k} N-\frac{1}{2} 3^{k-1} N-1=3^{k-1} N-1 .
$$

Thus

$$
\sum_{j} m_{k}\left(\varphi_{-}\left(\lambda_{j}^{(k-1)}\right)\right)+m_{k}\left(\varphi_{+}\left(\lambda_{j}^{(k-1)}\right)\right)=2 \cdot 3^{k-1} N-2 .
$$

To this we add $m_{k}(0), m_{k}(3)=\frac{1}{2} 3^{k-1} N$ and $m_{k}(6)=\frac{1}{2} 3^{k} N$ to obtain a total of $4 \cdot 3^{k-1} N-1$ for the sum of multiplicities of all eigenvalues of $\Gamma_{k}$ except 5 . Thus $m_{k}(5)=\frac{1}{2} 3^{k+1} N-\left(4 \cdot 3^{k-1} N-1\right)=\frac{1}{2} 3^{k-1} N+1$.

The next part of the spectral decimation method is the fact that

$$
\operatorname{spectrum}(\mathcal{F})=\frac{3}{2} \lim _{k \rightarrow \infty} 5^{k} \operatorname{spectrum}\left(\Gamma_{k}\right)
$$


in the following precise sense. Let $\left\{\delta_{k}\right\}_{k>n}$ be a sequence of signs $\delta_{k}= \pm$ with all but a finite number being minus. If $\lambda_{n} \in \operatorname{spectrum}\left(\Gamma_{n}\right)$ with multiplicity $m_{n}\left(\lambda_{n}\right)$ and

$$
\lambda_{k}=\varphi_{\delta_{k}}\left(\lambda_{k-1}\right) \text { for all } k>n,
$$

then $\lambda_{k}$ is in $\operatorname{spectrum}\left(\Gamma_{k}\right)$ with the same multiplicity (except that when $\lambda_{n}=6$ we must choose $\delta_{n+1}=+$ so that $\lambda_{n+1}=3$ rather than 2). Also, the $\lambda_{k}$-eigenfunctions on $\Gamma_{k}$ are obtained from the $\lambda_{k-1}$-eigenfunctions on $\Gamma_{k-1}$ by (4.5). But now

$$
\lambda=\frac{3}{2} \lim _{k \rightarrow \infty} 5^{k} \lambda_{k}
$$

exists, and the sequence of $\lambda_{k}$-eigenfunctions on $\Gamma_{k}$ converges uniformly to a $\lambda$ eigenfunction on $\mathcal{F}$, giving an eigenspace of multiplicity $m_{n}\left(\lambda_{n}\right)$. (The existence of the limit (4.9) is a consequence of the requirement that $\delta_{k}$ is minus for all sufficiently large $k$.) Conversely, for every $\lambda \in \operatorname{spectrum}(\mathcal{F})$ there exists a smallest $n$, called the generation of birth, such that $\lambda$ is obtained in this way. We have four possibilities:

(i) $n=0$,

(ii) $n=1$ and $\lambda_{1}=2$,

(iii) $n \geq 1$ and $\lambda_{n}=5$,

(iv) $n \geq 1$ and $\lambda_{n}=6$.

These facts are proved in [FS] for SG, but the same proof works for fractafolds (note that the factor $3 / 2$ is omitted in the definition of the Laplacian used in [FS], so the analog of (4.9) in [FS] is also missing this factor).

We may now give an exact description of the spectrum of $\mathcal{F}$. Let

$$
\psi(x)=\frac{3}{2} \lim _{k \rightarrow \infty} 5^{k} \varphi_{-}^{(k)}(x)
$$

where $\varphi_{-}^{(k)}$ denotes the composition of $\varphi_{-}$with itself $k$ times. The lowest eigenvalue in the spectrum of $\mathcal{F}$ is zero, with multiplicity $m(0)=1$. After that, the distinct eigenvalues follow a cyclic pattern. Since by Theorem 4.2 the nonzero eigenvalues of $\Gamma_{1}$ are $\left\{\varphi_{-}\left(\mu_{1}\right), \ldots, \varphi_{-}\left(\mu_{N_{0}}\right), 2,3, \varphi_{+}\left(\mu_{N_{0}}\right), \ldots, \varphi_{+}\left(\mu_{1}\right), 5,6\right\}$, the lowest portion of the spectrum of $\mathcal{F}$ (choosing $\delta_{k}$ to be minus for $k \geq 1$ ) will be

$$
\begin{gathered}
C_{1}^{\prime}=\left\{\psi\left(\mu_{1}\right), \ldots, \psi\left(\mu_{N_{0}}\right), 5 \psi(2), 5 \psi(3), 5 \psi\left(\varphi_{+}\left(\mu_{N_{0}}\right)\right),\right. \\
\left., \ldots, 5 \psi\left(\varphi_{+}\left(\mu_{1}\right)\right), 5 \psi(5), 5^{2} \psi(3)\right\} .
\end{gathered}
$$

Another application of Theorem 4.2 gives the eigenvalues of $\Gamma_{2}$ as

$$
\begin{aligned}
& \left\{\varphi_{-} \varphi_{-}\left(\mu_{1}\right), \ldots, \varphi_{-} \varphi_{-}\left(\mu_{N_{0}}\right), \varphi_{-}(2), \varphi_{-}(3), \varphi_{-} \varphi_{+}\left(\mu_{N_{0}}\right), \ldots,\right. \\
& \varphi_{-} \varphi_{+}\left(\mu_{1}\right), \varphi_{-}(5), 3, \varphi_{+}(5), \varphi_{+} \varphi_{+}\left(\mu_{1}\right), \ldots, \varphi_{+} \varphi_{+}\left(\mu_{N_{0}}\right), \varphi_{+}(3), \\
& \left.\varphi_{+}(2), \varphi_{+} \varphi_{-}\left(\mu_{N_{0}}\right), \ldots, \varphi_{+} \varphi_{-}\left(\mu_{1}\right), 5,6\right\} .
\end{aligned}
$$

This means that following $C_{1}^{\prime}$ in the spectrum of $\mathcal{F}$ will be

$$
\begin{aligned}
C_{1}^{\prime \prime}=\left\{5^{2} \psi\left(\varphi_{+}(5)\right), 5^{2} \psi\left(\varphi_{+} \varphi_{+}\left(\mu_{1}\right)\right), \ldots, 5^{2} \psi\left(\varphi_{+} \varphi_{+}\left(\mu_{N_{0}}\right)\right),\right. & \\
& 5^{2} \psi\left(\varphi_{+}(3)\right), 5^{2} \psi\left(\varphi_{+}(2)\right), 5^{2} \psi\left(\varphi_{+} \varphi_{-}\left(\mu_{N_{0}}\right)\right), \ldots \\
& \left.5^{2} \psi\left(\varphi_{+} \varphi_{-}\left(\mu_{1}\right)\right), 5^{2} \psi(5), 5^{3} \psi(3)\right\}
\end{aligned}
$$


The first cycle in the spectrum of $\mathcal{F}$ is

$$
C_{1}=\left\{C_{1}^{\prime}, C_{1}^{\prime \prime}, 5^{3} \psi\left(\varphi_{+}(5)\right)\right\}
$$

where the last eigenvalue is the lowest eigenvalue with generation of birth three. Note that aside from the highest three eigenvalues of $C_{1}$, the order is essentially palindromic. This means that the basic format of $C_{1}$ gets repeated as we go up the spectrum, with the powers of 5 and the iteration of $\varphi_{+}$and $\varphi_{-}$changing.

To describe the cycle $C_{k}$ for $k \geq 2$ let $2^{n-3}<k \leq 2^{n-2}$ determine $n$. Then there are sequences $\alpha$ and $\beta$ of length $n-1$ and sequences $\delta, \delta^{\prime}$ of length $\leq n-2$ of symbols + and - , all beginning with + and such that $\alpha=(\gamma,-)$ and $\beta=(\gamma,+)$ if $k$ is odd and $\alpha=(\gamma,+)$ and $\beta=(\gamma,-)$ if $k$ is even. We then have

$$
\begin{aligned}
C_{k}=\left\{5^{n}\right. & \psi\left(\varphi_{\alpha} \varphi_{-}\left(\mu_{1}\right)\right), \ldots, 5^{n} \psi\left(\varphi_{\alpha} \varphi_{-}\left(\mu_{N_{0}}\right)\right), 5^{n} \psi\left(\varphi_{\alpha}(2)\right), \\
& 5^{n} \psi\left(\varphi_{\alpha}(3)\right), 5^{n} \psi\left(\varphi_{\alpha} \varphi_{+}\left(\mu_{N_{0}}\right)\right), \ldots, 5^{n} \psi\left(\varphi_{\alpha} \varphi_{+}\left(\mu_{1}\right)\right), \\
& \left.5^{n} \psi\left(\varphi_{\alpha}(5)\right)\right), 5^{n} \psi\left(\varphi_{\gamma}(3)\right), 5^{n} \psi\left(\varphi_{\beta}(5)\right), 5^{n} \psi\left(\varphi_{\beta} \varphi_{+}\left(\mu_{1}\right)\right), \\
& \ldots, 5^{n} \psi\left(\varphi_{\beta} \varphi_{+}\left(\mu_{N_{0}}\right)\right), 5^{n} \psi\left(\varphi_{\beta}(3)\right), 5^{n} \psi\left(\varphi_{\beta}(2)\right) \\
& 5^{n} \psi\left(\varphi_{\beta} \varphi_{-}\left(\mu_{N_{0}}\right)\right), \ldots, 5^{n} \psi\left(\varphi_{\beta} \varphi_{-}\left(\mu_{1}\right)\right), 5^{n} \psi\left(\varphi_{\delta}(5)\right), \\
& \left.5^{n} \psi\left(\varphi_{\delta}(3)\right), 5^{n}\left(\varphi_{\delta^{\prime}}(5)\right)\right\},
\end{aligned}
$$

where $\varphi_{\alpha}=\varphi_{\alpha_{1}} \cdots \varphi_{\alpha_{n-1}}$, etc. In the case $k=2^{n-2}$ the last two eigenvalues have a factor $5^{n+1}$ rather than $5^{n}$. It is possible to give a precise description of $\gamma, \delta, \delta^{\prime}$ in terms of $k$, but it is rather complicated, so we omit it.

The eigenvalues $5^{n} \psi\left(\varphi_{\alpha} \varphi_{ \pm}\left(\mu_{j}\right)\right)$ and $5^{n} \psi\left(\varphi_{\beta} \varphi_{ \pm}\left(\mu_{j}\right)\right)$ have generation of birth zero, and hence multiplicity $m_{G}\left(\mu_{j}\right)$. The eigenvalues $5^{n} \psi\left(\varphi_{\alpha}(3)\right)$ and $5^{n} \psi\left(\varphi_{\beta}(3)\right)$ also have generation of birth zero, and hence multiplicity $m_{0}(6)$. The eigenvalues $5^{n} \psi\left(\varphi_{\alpha}(2)\right)$ and $5^{n} \psi\left(\varphi_{\beta}(2)\right)$ have generation of birth one and multiplicity one (if $G$ is 2-colorable). The eigenvalues $5^{n} \psi\left(\varphi_{\alpha}(5)\right)$ and $5^{n} \psi\left(\varphi_{\beta}(5)\right)$ have generation of birth one and multiplicity $m_{1}(5)=\frac{1}{2} N+1$, while $5^{n} \psi\left(\varphi_{\gamma}(3)\right)$ has generation of birth one and multiplicity $m_{1}(6)=\frac{3}{2} N$. This accounts for the multiplicities of all but the last three eigenvalues of the cycle, and these multiplicities are the same for all cycles.

The generation of birth of the last three eigenvalues is $j+2$ where $k=$ $2^{j}(2 \ell+1)$. The multiplicities of eigenvalues $5^{n} \psi\left(\varphi_{\delta}(5)\right)$ and $5^{n} \psi\left(\varphi_{\delta^{\prime}}(5)\right)$ are $m_{j+2}(5)$ $=\frac{1}{2} 3^{j+1} N+1$, while the multiplicity of eigenvalue $5^{n} \psi\left(\varphi_{\delta}(3)\right)$ is $m_{j+2}(6)=$ $\frac{1}{2} 3^{j+2} N$. These multiplicities vary from cycle to cycle, grow without bound, and depend only on $N$. That is, only the size of $\mathcal{F}$ counts for these multiplicities. These are the eigenvalues that really dominate the spectrum. If we consider all the other eigenvalues in all cycles $C_{j}$ for $j \leq 2^{n-2}$, their total multiplicities add up to a multiple of $2^{n}$, whereas there are on the order of $3^{n}$ total multiplicities in this portion of the spectrum. We may say that asymptotically almost all of the spectrum comes from the top three eigenvalues of the cycles, and depends only on the size of $\mathcal{F}$.

We summarize the above discussion as follows:

Theorem 4.3. The distinct eigenvalues of $\mathcal{F}$ are given in increasing order as

$$
\left\{0, C_{1}, C_{2}, \ldots\right\}
$$




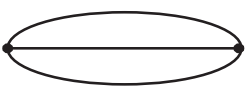

0,6

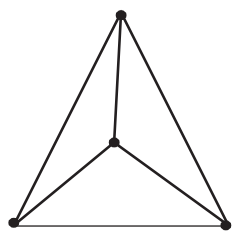

$0,4_{3}$

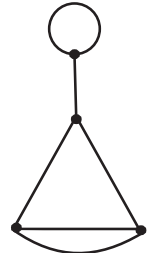

$0,1,4,5$
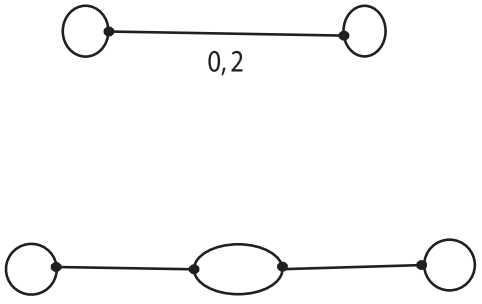

$0,3-\sqrt{5}, 2,3+\sqrt{5}$

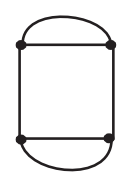

$0,2,4,6$

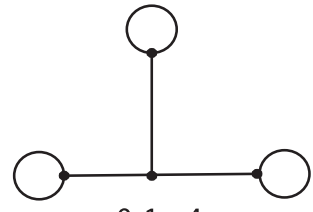

$0,12,4$

Figure 4.1. The cell graph $G$ and eigenvalues for all examples with $N=2$ or 4 .

where $C_{1}$ is given by (4.13) and $C_{k}$ for $k \geq 2$ by (4.14). The multiplicities of the eigenvalues in cycle $C_{k}$ are given by

$$
\begin{aligned}
& \left\{m_{G}\left(\mu_{1}\right), \ldots, m_{G}\left(\mu_{N_{0}}\right), 1, m_{0}(6), m_{G}\left(\mu_{N_{0}}\right), \ldots, m_{G}\left(\mu_{1}\right), \frac{1}{2} N+1,\right. \\
& \quad \frac{3}{2} N, \frac{1}{2} N+1, m_{G}\left(\mu_{1}\right), \ldots, m_{G}\left(\mu_{N_{0}}\right), m_{0}(6), 1, m_{G}\left(\mu_{N_{0}}\right), \\
& \left.\quad \ldots, m_{G}\left(\mu_{1}\right), \frac{1}{2} 3^{j+1} N+1, \frac{1}{2} 3^{j+2} N, \frac{1}{2} 3^{j+1} N+1\right\},
\end{aligned}
$$

where $k=2^{j}(2 \ell+1)$. In particular, the spectrum is uniquely determined by the spectrum of $G$, and asymptotically almost all of the spectrum depends only on $N$.

In Figure 4.1 we show the cell graph $G$ for all possible fractafolds with $N=2$ or 4 , together with a list of eigenvalues (subscripts indicate multiplicities greater than one).

We end this section by giving an explicit description of a pair of fractafolds that are isospectral but not homeomorphic. To show that they are isospectral we need to show that their cell graphs are isospectral. To show that they are not homeomorphic we need to show that after consolidating all triangles, the two graphs remain distinct. There are many explicit examples of isospectral pairs of graphs in the literature, but apparently none that are 3-regular. Robert Brooks [Br3] has indicated how to modify 4-regular isospectral graphs of Sunada type by a substitution scheme to obtain 3-regular pairs (see [Br1], [B] where related ideas are discussed). By starting with an example $([\mathrm{Bu},[\mathrm{Br} 1)$ of an isospectral pair of 4-regular graphs with seven vertices, based on the group $S L(3,2)=P S L(2,7)$, and substituting a 2-vertex graph for each vertex, we obtain the isospectral pair of 3 -regular graphs with fourteen vertices each shown in Figure 4.2. The fractafolds 


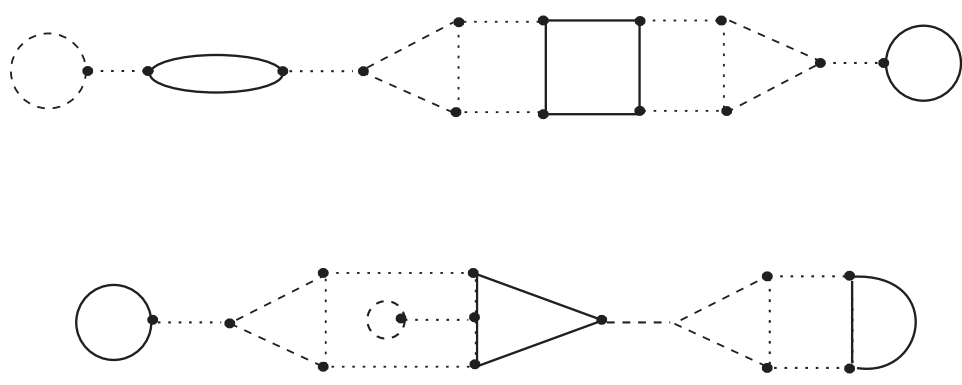

FiguRE 4.2. An isospectral pair of 3-regular graphs with fourteen vertices. Three different types of edges are indicated.

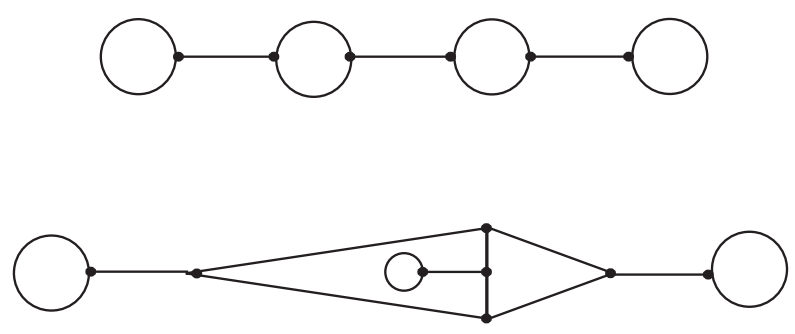

FiguRE 4.3. The above graphs after consolidation of all triangles.

with these cell graphs are isospectral. After consolidating all triangles we obtain the graphs shown in Figure 4.3, which are clearly not isomorphic, as they have different numbers of vertices. Thus the fractafolds are not homeomorphic.

We give a brief explanation why the graphs in Figure 4.2 are isospectral. The basic principle is that $k$-regular graphs are isospectral if and only if they have the same "length spectrum", the number of distinct closed paths of length $m$ (for $m \leq N)$. With the correct counting convention this number is just $\operatorname{tr} A^{m}$, where $A$ is the incidence matrix of the graph, and

$$
\operatorname{tr} A^{m}=\sum_{j=1}^{N}\left(\widetilde{\lambda}_{j}\right)^{m}
$$

where $\left\{\widetilde{\lambda}_{j}\right\}$ is the spectrum of $A$ (just an affine image of the spectrum of the Laplacian). Knowing all the quantities (4.17) for $m \leq N$ determines all the elementary symmetric polynomials in the eigenvalues up to order $N$, hence the characteristic polynomial of $A$, hence the eigenvalues.

The Sunada construction of isospectral graphs yields even more information. The edges of the graphs are colored, and the number of closed paths of length $m$ with a given coloring scheme is identical for both graphs. The coloring of edges in Figure 4.2 is indicated by the use of solid, dashed and dotted lines. The solid and dashed lines come from the 4-regular graphs (just consolidate pairs of vertices in Figure 4.2 along dotted lines), which have this Sunada property, and it is easy to see that the Sunada property is inherited in the substitution process. 


\section{FRACTAFOLDS WITH BOUNDARY}

If $\mathcal{F}$ is a compact fractafold with boundary based on SG, we create the double $\widetilde{\mathcal{F}}$ by taking two copies of $\mathcal{F}$ and identifying corresponding boundary points. If $G$ is a cell graph for $\mathcal{F}$, we see that the corresponding cell graph $\widetilde{G}$ for $\widetilde{\mathcal{F}}$ is obtained by taking two copies of $G$ and creating one or two edges joining corresponding vertices that have fewer than three edges, so that $\widetilde{G}$ becomes 3-regular. Let $R$ denote the reflection on $\widetilde{\mathcal{F}}$ that interchanges corresponding points in the two copies of $\mathcal{F}$, and similarly let $r$ denote the corresponding reflection on $\widetilde{G}$. We can then define odd and even functions on $\widetilde{\mathcal{F}}$ or $\widetilde{G}$ with respect to these reflections, and odd and even extensions from functions on $\mathcal{F}$ or $G$ to functions on $\widetilde{\mathcal{F}}$ or $\widetilde{G}$.

Now it is easy to see the reflection $R$ preserves eigenspaces on $\widetilde{\mathcal{F}}$, and so eigenspaces split into odd and even functions. Moreover, upon restriction to $\mathcal{F}$, the odd functions satisfy Dirichlet boundary conditions, and the even functions satisfy Neumann boundary conditions. Conversely, Dirichlet eigenfunctions on $\mathcal{F}$ extend by odd reflection to odd eigenfunctions on $\widetilde{\mathcal{F}}$, and Neumann eigenfunctions on $\mathcal{F}$ extend by even reflection to even eigenfunctions on $\widetilde{\mathcal{F}}$. Thus the problem of finding the Dirichlet and Neumann spectra on $\mathcal{F}$ becomes merely a matter of sorting the spectrum of $\widetilde{\mathcal{F}}$ into even and odd parts. We denote multiplicities of odd eigenvalues with a prime, and even eigenvalues with a double prime.

The sorting is quite straightforward. The graph $\widetilde{G}$ has $N$ odd and $N$ even eigenfunctions, where $N$ is the number of vertices of $G$. Of course the 0-eigenfunction is even. Note that $\widetilde{G}$ is 2-colorable if and only if $G$ is 2-colorable, in which case the 6 -eigenfunction is odd. Thus the odd spectrum of $\widetilde{G}$ has distinct eigenvalues $\left\{\mu_{1}^{\prime}, \ldots, \mu_{N_{1}}^{\prime}, 6\right\}$, with multiplicities $m_{G}^{\prime}\left(\mu_{j}^{\prime}\right)$ satisfying

$$
\sum_{j=1}^{N_{1}} m_{G}^{\prime}\left(\mu_{j}^{\prime}\right)= \begin{cases}N-1 & \text { if } G \text { is 2-colorable } \\ N & \text { if } G \text { is not 2-colorable }\end{cases}
$$

and

$$
m_{G}^{\prime}(6)=\left\{\begin{array}{l}
1 \text { if } G \text { is 2-colorable } \\
0 \text { if } G \text { is not 2-colorable }
\end{array}\right.
$$

while the even spectrum has distinct eigenvalues $\left\{0, \mu_{1}^{\prime \prime}, \ldots, \mu_{N_{2}}^{\prime \prime}\right\}$ with multiplicities $m_{G}^{\prime \prime}\left(\mu_{j}^{\prime \prime}\right)$ satisfying

$$
\sum_{j=1}^{N_{2}} m_{G}^{\prime \prime}\left(\mu_{j}^{\prime \prime}\right)=N-1
$$

and $\left\{\mu_{1}^{\prime}, \ldots, \mu_{N_{1}}^{\prime}\right\} \cup\left\{\mu_{1}^{\prime \prime}, \ldots, \mu_{N_{2}}^{\prime \prime}\right\}=\left\{\mu_{1}, \ldots, \mu_{N_{0}}\right\}$. When we sort the eigenfunctions (with $\lambda \neq 6$ ) on $\widetilde{\Gamma}_{0}$ into odd and even, we obtain the same eigenvalues and multiplicities. We may then deduce the multiplicities of the odd and even 6-eigenspaces by a counting argument. Since $\widetilde{G}$ has $2 N$ vertices, $\widetilde{\Gamma}_{0}$ has $3 N$ vertices. Let $M$ denote the number of these that correspond to boundary points of $\mathcal{F}$. Note that $M$ and $N$ must have the same parity. Then $\frac{1}{2}(3 N-M)$ and $\frac{1}{2}(3 N+M)$ are the 
dimensions of odd and even functions on $\widetilde{\Gamma}_{0}$. By (5.1) and (5.2) this means

$$
\begin{cases}m_{0}^{\prime}(6)= \begin{cases}\frac{1}{2}(N-M)+1 & \text { if } G \text { is 2-colorable } \\ \frac{1}{2}(N-M) & \text { if } G \text { is not 2-colorable }\end{cases} \\ m_{0}^{\prime \prime}(6)=\frac{1}{2}(N+M)\end{cases}
$$

for the multiplicities of the odd and even 6-eigenvalue. This completes the sorting of all eigenvalues with generation of birth equal to zero. It is also clear, in the 2-colorable case, that the 2-eigenfunction of $\widetilde{\Gamma}_{1}$ is odd.

It remains to sort the eigenvalues with higher generation of birth, namely $\lambda=5$ and $\lambda=6$ born in $\widetilde{\Gamma}_{k}$. For $\lambda=6$ the answer is easy, since $\widetilde{\Gamma}_{k-1}$ has $3^{k} N$ vertices and $M$ of them correspond to boundary vertices of $\mathcal{F}$. Each 6 -eigenfunction of the standard basis is associated to a vertex of $\widetilde{\Gamma}_{k-1}$. Now the boundary vertices yield even eigenfunctions. For nonboundary vertices, each pair (the corresponding vertices of the two copies of $\Gamma_{k-1}$ ) yields one odd and one even eigenfunction. Thus

$$
\left\{\begin{array}{l}
m_{k}^{\prime}(6)=\frac{1}{2}\left(3^{k} N-M\right) \\
m_{k}^{\prime \prime}(6)=\frac{1}{2}\left(3^{k} N+M\right),
\end{array}\right.
$$

and of course $m_{k}^{\prime}(3)=m_{k-1}^{\prime}(6)$ and $m_{k}^{\prime \prime}(3)=m_{k-1}^{\prime \prime}(6)$. For $\lambda=5$ we resort once again to counting. The odd multiplicities must sum to $\frac{1}{2}\left(3^{k+1} N-M\right)$ and the even multiplicities to $\frac{1}{2}\left(3^{k+1} N+M\right)$. Assuming this is the case for $\widetilde{\Gamma}_{k-1}$, we count $4 \cdot 3^{k-1} N-M$ odd and $4 \cdot 3^{k-1} N+M-1$ even total from Theorem 4.2 for all eigenvaleus except $\lambda=5$; hence

$$
\left\{\begin{array}{l}
m_{k}^{\prime}(5)=\frac{1}{2}\left(3^{k-1} N+M\right), \\
m_{k}^{\prime \prime}(5)=\frac{1}{2}\left(3^{k-1} N-M\right)+1 .
\end{array}\right.
$$

Theorem 5.1. For a compact fractafold with boundary, the distinct Dirichlet eigenvalues in increasing order have cyclic structure

$$
\left\{C_{1}^{\prime}, C_{2}^{\prime}, \ldots\right\}
$$

where

$$
\begin{aligned}
& C_{k}^{\prime}=\left\{5^{n} \psi\left(\varphi_{\alpha} \varphi_{-}\left(\mu_{1}\right)\right), \ldots, 5^{n} \psi\left(\varphi_{\alpha} \varphi_{-}\left(\mu_{N_{1}}^{\prime}\right)\right), 5^{n} \psi\left(\varphi_{\alpha}(2)\right),\right. \\
& 5^{n} \psi\left(\varphi_{\alpha}(3)\right), 5^{n} \psi\left(\varphi_{\alpha} \varphi_{+}\left(\mu_{N_{1}}^{\prime}\right)\right), \ldots, 5^{n} \psi\left(\varphi_{\alpha} \varphi_{+}\left(\mu_{1}^{\prime}\right)\right), \\
& 5^{n} \psi\left(\varphi_{\alpha}(5)\right), 5^{n} \psi\left(\varphi_{\gamma}(3)\right), 5^{n} \psi\left(\varphi_{\beta}(5)\right), 5^{n} \psi\left(\varphi_{\beta} \varphi_{+}\left(\mu_{1}^{\prime}\right)\right), \\
& \ldots, 5^{n} \psi\left(\varphi_{\beta} \varphi_{+}\left(\mu_{N_{1}}^{\prime}\right)\right), 5^{n} \psi\left(\varphi_{\beta}(3)\right), 5^{n} \psi\left(\varphi_{\beta}(2)\right), \\
& 5^{n} \psi\left(\varphi_{\beta} \varphi_{-}\left(\mu_{N_{1}}^{\prime}\right)\right), \ldots, 5^{n} \psi\left(\varphi_{\beta} \varphi_{-}\left(\mu_{1}^{\prime}\right)\right), 5^{n} \psi\left(\varphi_{\delta}(5)\right), \\
&\left.5^{n} \psi\left(\varphi_{\delta}(3)\right), 5^{n} \psi\left(\varphi_{\delta^{\prime}}(5)\right)\right\},
\end{aligned}
$$

and corresponding multiplicities

$$
\begin{aligned}
& \left\{m_{G}^{\prime}\left(\mu_{1}^{\prime}\right), \ldots, m_{G}^{\prime}\left(\mu_{N_{1}}^{\prime}\right), 1, m_{0}^{\prime}(6), m_{G}^{\prime}\left(\mu_{N_{1}}^{\prime}\right), \ldots, m_{G}^{\prime}\left(\mu_{1}^{\prime}\right),\right. \\
& \quad \frac{1}{2}(N+M), \frac{1}{2}(3 N-M), \frac{1}{2}(N+M), m_{G}^{\prime}\left(\mu_{1}^{\prime}\right), \ldots, m_{G}^{\prime}\left(\mu_{N_{1}}^{\prime}\right), \\
& \quad m_{0}^{\prime}(6), 1, m_{G}^{\prime}\left(\mu_{N_{1}}^{\prime}\right), \ldots, m_{G}^{\prime}\left(\mu_{1}^{\prime}\right), \frac{1}{2}\left(3^{j+1} N+M\right), \\
& \left.\quad \frac{1}{2}\left(3^{j+2} N-M\right), \frac{1}{2}\left(3^{j+1} N+M\right)\right\} .
\end{aligned}
$$


Similarly, the Neumann eigenvalues in increasing order are

$$
\left\{0, C_{1}^{\prime \prime}, C_{2}^{\prime \prime}, \ldots\right\}
$$

with

$$
\begin{aligned}
C_{k}^{\prime \prime}=\left\{5^{n} \psi\left(\varphi_{\alpha} \varphi_{-}\left(\mu_{1}^{\prime \prime}\right)\right), \ldots, 5^{n} \psi\left(\varphi_{\alpha} \varphi_{-}\left(\mu_{N_{2}}^{\prime \prime}\right)\right), 5^{n} \psi\left(\varphi_{\alpha}(3)\right),\right. & 5^{n} \psi\left(\varphi_{\alpha} \varphi_{+}\left(\mu_{N_{2}}^{\prime \prime}\right)\right), \ldots, 5^{n} \psi\left(\varphi_{\alpha} \varphi_{+}\left(\mu_{1}^{\prime \prime}\right)\right), 5^{n} \psi\left(\varphi_{\alpha}(5)\right), \\
& 5^{n} \psi\left(\varphi_{\gamma}(3)\right), 5^{n} \psi\left(\varphi_{\beta}(5)\right), 5^{n} \psi\left(\varphi_{\beta} \varphi_{+}\left(\mu_{1}^{\prime \prime}\right)\right), \ldots, \\
& 5 \psi\left(\varphi_{\beta} \varphi_{+}\left(\mu_{N_{2}}^{\prime \prime}\right)\right), 5^{n} \psi\left(\varphi_{\beta}(3)\right), 5^{n} \psi\left(\varphi_{\beta} \varphi_{-}\left(\mu_{N_{2}}^{\prime \prime}\right)\right), \ldots, \\
& \left.5^{n} \psi\left(\varphi_{\beta} \varphi_{-}\left(\mu_{1}^{\prime \prime}\right)\right), 5^{n} \psi\left(\varphi_{\delta}(5)\right), 5^{n} \psi\left(\varphi_{\delta}(3)\right), 5^{n} \psi\left(\varphi_{\delta^{\prime}}(5)\right)\right\},
\end{aligned}
$$

and corresponding multiplicities

$$
\begin{gathered}
\left\{m_{G}^{\prime \prime}\left(\mu_{1}^{\prime \prime}\right), \ldots, m_{G}^{\prime \prime}\left(\mu_{N_{2}}^{\prime \prime}\right), \frac{1}{2}(N+M), m_{G}^{\prime \prime}\left(\mu_{N_{2}}^{\prime \prime}\right), \ldots, m_{G}^{\prime \prime}\left(\mu_{1}^{\prime \prime}\right),\right. \\
\quad \frac{1}{2}(N-M)+1, \frac{1}{2}(3 N+M), \frac{1}{2}(N-M)+1, m_{G}^{\prime \prime}\left(\mu_{1}^{\prime \prime}\right), \\
\left.\quad \ldots, m_{G}^{\prime \prime}\left(\mu_{N_{2}}^{\prime \prime}\right)\right), \frac{1}{2}(N+M), m_{G}^{\prime \prime}\left(\mu_{N_{2}}^{\prime \prime}\right), \ldots, m_{G}^{\prime \prime}\left(\mu_{1}^{\prime \prime}\right), \\
\left.\frac{1}{2}\left(3^{j+1} N-M\right)+1, \frac{1}{2}\left(3^{j+2} N+M\right), \frac{1}{2}\left(3^{j+1} N-M\right)+1\right\} .
\end{gathered}
$$

The notation here is the same as in Theorem 4.3, with the same modifications when $k$ is a power of 2 . Also, in some cases the multiplicities $m_{0}^{\prime}(6), \frac{1}{2}(3 N-M)$, or $\frac{1}{2}(N-M)+1$ in (5.8) or (5.11) may be zero, in which case the corresponding eigenvalues should be deleted from (5.7) or (5.10). Asymptotically, almost all of the spectra are the same, and depend only on $N$.

As an example, consider the original SG. Here $N=1$ and $M=3$, and $\widetilde{G}$ consists of two vertices with three edges joining them, with eigenvalues 0 and 6 . In this case $N_{1}=N_{2}=0$, but $G$ is 2 -colorable. Also $m_{0}^{\prime}(6), \frac{1}{2}(3 N-M)$ and $\frac{1}{2}(N-M)+1$ all vanish. Thus the Dirichlet cycle consists of just seven eigenvalues,

$$
\begin{aligned}
& C_{k}^{\prime}=\left\{5^{n} \psi\left(\varphi_{\alpha}(2)\right), 5^{n} \psi\left(\varphi_{\alpha}(5)\right), 5^{n} \psi\left(\varphi_{\beta}(5)\right), 5^{n} \psi\left(\varphi_{\beta}(2)\right), 5^{n} \psi\left(\varphi_{\delta}(5)\right),\right. \\
&\left.5^{n} \psi\left(\varphi_{\delta}(3)\right), 5^{n} \psi\left(\varphi_{\delta^{\prime}}(5)\right)\right\}
\end{aligned}
$$

with multiplicities $\left\{1,2,2,1, \frac{1}{2}\left(3^{j+1}+3\right), \frac{1}{2}\left(3^{j+2}-3\right), \frac{1}{2}\left(3^{j+1}+3\right)\right\}$. This is the "octave structure" first noted explicitly in [GRS. The Neumann cycle consists of six eigenvalues,

$C_{k}^{\prime \prime}=\left\{5^{n} \psi\left(\varphi_{\alpha}(3)\right), 5^{n} \psi\left(\varphi_{\gamma}(3)\right), 5^{n} \psi\left(\varphi_{\beta}(3)\right), 5^{n} \psi\left(\varphi_{\delta}(5)\right), 5^{n} \psi\left(\varphi_{\delta}(3)\right), 5^{n} \psi\left(\varphi_{\delta^{\prime}}(5)\right)\right\}$

with multiplicities $\left\{2,3,2, \frac{1}{2}\left(3^{j+1}-1\right), \frac{1}{2}\left(3^{j+2}+3\right), \frac{1}{2}\left(3^{j+1}-1\right)\right\}$. These results are consistent with [FS]. Note that the cycles $C_{k}^{\prime}$ and $C_{k}^{\prime \prime}$ of the two spectra have three eigenvalues in common, namely the last three. In fact the Dirichlet eigenspace contains the Neumann eigenspace for the $5^{n} \psi\left(\varphi_{\delta}(5)\right)$ and $5^{n} \psi\left(\varphi_{\delta^{\prime}}(5)\right)$ eigenvalues, and the Neumann eigenspace contains the Dirichlet eigenspace for the $5^{n} \psi\left(\varphi_{\delta}(3)\right)$ eigenvalue. The common eigenspaces are called "prelocalized" eigenfunctions in [BK].

\section{General metrics}

The Laplacian on a manifold depends on the choice of a Riemannian metric. The Laplacian on a fractafold $\mathcal{F}$ with a given cellular construction that we have studied so far in this paper may be thought of as arising from a "standard metric" that treats all the cells in the cellular construction as having the same "size". This is implicit in (3.1), (3.2) and (3.6). In this section we will discuss briefly what happens 
if we consider more general Laplacians associated to "metrics" that allow the cells to have different "sizes". Actually, we will see that there are two independent parameters, which we will denote by $a$ and $b$, that measure the size of a cell in terms of energy and measure. For simplicity we deal only with compact fractafolds without boundary.

Note that every edge in each graph $\Gamma_{k}$ lies entirely in one cell, even though the vertices may belong to two different cells. Thus the sum in (3.1) may be broken up into $N$ separate sums, each giving the contribution from a single cell. If we are given positive weights $\left\{a_{i}\right\}$ for the cells, we multiply the energy from cell $K_{i}$ by $a_{i}$ and sum, to obtain

$$
E_{k}^{(a)}(u, u)=\sum_{i=1}^{N} a_{i} \sum_{e_{i k}}|u(x)-u(y)|^{2},
$$

where $e_{i k}$ denotes the set of edges on $\Gamma_{k}$ lying in $K_{i}$. We may then define $\mathcal{E}_{k}^{(a)}$ and $\mathcal{E}^{(a)}$ by the analog of (3.3) and (3.4). This is merely

$$
\mathcal{E}^{(a)}(u, u)=\sum_{i=1}^{N} a_{i} \mathcal{E}\left(\left.u\right|_{K_{i}},\left.u\right|_{K_{i}}\right) .
$$

We may define a Laplacian associated with $\mathcal{E}^{(a)}$ and any reasonable measure $\mu$ by using the weak formulation (3.7). We will only consider a special class of measures. Let $\mu_{i}$ denote the standard measure on $K_{i}\left(\right.$ with $\mu_{i}\left(K_{i}\right)=1$ ) and let

$$
\mu^{(b)}=\sum_{i=1}^{N} b_{i} \mu_{i}
$$

for some choice of positive weights $\left\{b_{i}\right\}$. In other words,

$$
\mu^{(b)}(A)=\sum_{i=1}^{N} b_{i} \mu_{i}\left(A \cap K_{i}\right)
$$

Then define $\Delta^{(a, b)}$ as follows: for $u \in \operatorname{dom} \mathcal{E}^{(a)}$ and $f$ continuous, $u \in \operatorname{dom} \Delta^{(a, b)}$ and $\Delta^{(a, b)} u=f$ means

$$
-\mathcal{E}^{(a)}(u, v)=\int f v d \mu^{(b)} \text { for all } v \in \operatorname{dom} \mathcal{E}^{(a)} .
$$

If $u$ is supported in the interior of a cell $K_{i}$, then

$$
\Delta^{(a, b)} u=a_{i} b_{i}^{-1} \Delta u \text {. }
$$

However, this is not the whole story, as $\Delta^{(a, b)}$ depends on both sets of parameters, not just the quotients. What is going on is that $\Delta^{(a, b)}$ is constructed by gluing together the operators $a_{i} b_{i}^{-1} \Delta$ on $K_{i}$ using a matching rule that depends on $\left\{a_{i}\right\}$. More precisely, let $\left\{u_{i}\right\}$ and $\left\{f_{i}\right\}$ be defined on $K_{i}$ and satisfy $a_{i} b_{i}^{-1} \Delta u_{i}=f_{i}$ in the sense of the standard Laplacian on $K_{i}$, and suppose $u_{i}$ and $f_{i}$ are well defined at all terminal points, $u_{i}(x)=u_{i^{\prime}}(x)$ and $f_{i}(x)=f_{i^{\prime}}(x)$ if $x$ is a terminal point of both $K_{i}$ and $K_{i^{\prime}}$ identified in $\mathcal{F}$. Then $\Delta^{(a, b)} u=f$ if and only if

$$
a_{i} \partial_{n} u_{i}(x)+a_{i^{\prime}} \partial_{n} u_{i^{\prime}}(x)=0
$$

at all identified terminal points. 
It is also possible to give a pointwise formula for $\Delta^{(a, b)} u$. At interior junction points of $K_{i}$ we just multiply (3.6) by $a_{i} b_{i}^{-1}$. At an identified terminal point $x$ as above we have

$$
\begin{aligned}
\Delta^{(a, b)} u(x)=\lim _{k \rightarrow \infty} \frac{3 \cdot 5^{k}}{b_{i}+b_{i^{\prime}}} & \left(a_{i}\left(2 u(x)-u\left(y_{k}\right)-u\left(z_{k}\right)\right)\right. \\
& \left.+a_{i^{\prime}}\left(2 u(x)-u\left(y_{k}^{\prime}\right)-u\left(z_{k}^{\prime}\right)\right)\right)
\end{aligned}
$$

where $y_{k}, z_{k}$ and $y_{k}^{\prime}, z_{k}^{\prime}$ are the neighbors of $x$ on level $k$ in $K_{i}$ and $K_{i^{\prime}}$.

It is easy to see that if we split a cell $K_{i}$ in a cellular construction into three cells $K_{i 1}, K_{i 2}, K_{i 3}$ of the next generation, then the $a_{i}$ factor must be replaced by $\frac{5}{3} a_{i}$, and the $b_{i}$ factor must be replaced by $\frac{1}{3} b_{i}$ in order to obtain the same Laplacian.

Each Laplacian $\Delta^{(a, b)}$ has a distinct discrete spectrum, which may in principle be described as follows. To decide if $\lambda$ is an eigenvalue, we consider all possible solutions of

$$
\Delta u_{i}=a_{i}^{-1} b_{i} \lambda u_{i} \text { on } K_{i}
$$

and try to glue them together to get a continuous function on $\mathcal{F}$ satisfying the matching conditions (6.7). For a generic choice of $\lambda$, the solutions of (6.9) are uniquely determined by the values of $u_{i}$ at the terminal points of $K_{i}$. That means that we need to specify the values of $u$ at the $\frac{3}{2} N$ junction points of level zero. The matching conditions (6.7) give us $\frac{3}{2} N$ homogeneous linear equations in these $\frac{3}{2} N$ parameters, and so generically there will be no nontrivial solutions. But for special choices of $\lambda$, the determinant of this linear system will vanish and nontrivial solutions will exist. In fact it is possible to describe explicitly how the normal derivatives that enter into (6.7) are determined by the values at the terminal points of solutions of the eigenvalue equation (6.9), in terms of "special functions". We will not give the details here, except to note that the description is complicated enough that there is no hope of getting an explicit solution to the eigenvalue problem.

But there are also nongeneric choices of $\lambda$ that yield localized eigenfunctions supported on just one cell. In fact, one only has to choose one of the prelocalized eigenspaces described at the end of Section 5. The eigenvalues get multiplied by $a_{i} b_{i}^{-1}$. Thus the spectrum of $\mathcal{F}$ contains eigenvalues

$$
\left\{a_{i} b_{i}^{-1} 5^{n} \psi\left(\varphi_{\delta}(5)\right), a_{i} b_{i}^{-1} 5^{n} \psi\left(\varphi_{\delta}(3)\right), a_{i} b_{i}^{-1} 5^{n} \psi\left(\varphi_{\delta^{\prime}}(5)\right)\right\}
$$

with multiplicities

$$
\left\{\frac{1}{2}\left(3^{j+1}-1\right), \frac{1}{2}\left(3^{j+2}-3\right), \frac{1}{2}\left(3^{j+1}-1\right)\right\} .
$$

Again it seems likely that these eigenvalues comprise asymptotically almost all of the spectrum.

\section{REFERENCES}

[BR] C. Bandt and T. Retta, Topological spaces admitting a unique fractal structure, Fund. Math. 141 (1992), 257-268.' MR 94c:28009

[B] M. Barlow, Diffusion on fractals, Lecture Notes Math., vol.1690 (1998). MR 2000a:60148

[BK] M. Barlow and J. Kigami, Localized eigenfunctions of the Laplacian on p.c.f. self-similar sets, J. London Math. Soc. 56 (1997), 320-332. MR 99b:35162

[BP] M. Barlow and E. Perkins, Brownian motion on the Sierpinski gasket, Probab. Theory Related Fields 79 (1988), 543-623. MR 89g:60241

[Br1] R. Brooks, The Sunada method, Contemp. Math. 231 (1999), 25-35. MR 2000g:58049

[Br2] R. Brooks, Non-Sunada graphs, Ann. Inst. Fourier, Grenoble 49 (1999), 707-725. MR 2000i:58062 
[Br3] R. Brooks, personal communication.

$[\mathrm{Bu}]$ P. Buser, Cayley graphs and planar isospectral domains, Springer Lecture Notes Math. 1339 (1988), 64-77. MR 90a:58177.

[DSV] K. Dalrymple, R. Strichartz and J. Vinson, Fractal differential equations on the Sierpinski gasket, J. Fourier Anal. Appl. 5 (1999), 203-284. MR 2000k:31016

[DM] W. Debski and J. Mioduszewski, Simple plane images of the Sierpinski triangular curve are nowhere dense, Colloq. Math. 59 (1990), 125-140. MR 92e:54039

[F] K. Falconer, Fractal Geometry: Mathematical Foundations and Applications, John Wiley \& Sons, New York, 1990. MR 92j:28008

[F-TN] A. Figa-Talamanca and C. Nebbia, Harmonic analysis and representation theory for groups acting on homogeneous trees, London Math. Soc. Lecture Note Series 162 (1991). MR 93f:22004

[FS] M. Fukushima and T. Shima, On a spectral analysis for the Sierpinski gasket, Potential Anal. 1 (1992), 1-35. MR 95b:31009

[GRS] M. Gibbons, A. Raj and R. Strichartz, The finite element method on the Sierpinski gasket, Constructive Approx. 17 (2001), 561-588. MR 2002i:28010

[Ki1] J. Kigami, Analysis on Fractals, Cambridge University Press, 2001. MR 2002c:28015

[Ki2] J. Kigami, Harmonic analysis for resistance forms, J. Functional Anal., to appear.

[MT] L. Malozemov and A. Teplyaev, Self-similarity, operators and dynamics, preprint.

[MST] R. Meyers, R. Strichartz and A. Teplyaev, Dirichlet forms on the Sierpinski gasket, Pacific J. Math. (to appear).

[Sa] C. Sabot, Existence and uniqueness of diffusions on finitely ramified self-similar fractals, Ann. Scient. Ec. Norm. Sup. $4^{\text {eme }}$ série 30 (1997), 605-673. MR 98h:60118

[Sh1] T. Shima, On eigenvalue problems for the random walks on the Sierpinski pre-gaskets, Japan J. Indust. Appl. Math. 8 (1991), 127-141. MR 92g:60094

[Sh2] T. Shima, On eigenvalue problems for Laplacians on p.c.f. self-similar sets, Japan J. Indust. Appl. Math. 13 (1996), 1-23. MR 97f:28028

[Shi] T. Shirai, The spectrum of infinite regular line graphs, Trans. Amer. Math. Soc. 352 (1999), 115-132. MR 2001f:05092

[S1] R. Strichartz, Fractals in the large, Can. J. Math. 50 (1998), 638-657. MR 99f:28015

[S2] R. Strichartz, Analysis on fractals, Notices American Mathematical Society 46 (1999), 1199-1208. MR 2000i:58035

[T] A. Teplyaev, Spectral analysis on infinite Sierpinski gaskets, J. Functional Anal. 159 (1989), 537-567. MR 99j:35153

Department of Mathematics, Malott Hall, Cornell University, Ithaca, New York 14853

E-mail address: str@math.cornell.edu 